\title{
Calibration of a modified Johnson-Cook model using the Virtual Fields Method and a heterogeneous thermo-mechanical tensile test
}

\author{
J.M.P. Martins ${ }^{\mathrm{a}, \mathrm{b}, *}$, S. Thuillier ${ }^{\mathrm{b}}$, A. Andrade-Campos ${ }^{\mathrm{a}}$ \\ ${ }^{a}$ Centre for Mechanical Technology and Automation, Dep. Mechanical Engineering, University of Aveiro, Portugal \\ ${ }^{b}$ Univ. Bretagne Sud, UMR CNRS 6027, IRDL, F-56100 Lorient, France
}

\begin{abstract}
Classical calibration procedures for phenomenological thermo-elasto-viscoplastic constitutive models usually involve a large number of tests to identify all the material parameters, leading to long experimental campaigns. In the present work, a novel calibration methodology that takes full advantage of full-field measurements of a heterogeneous test is proposed. It relies on an innovative combination of the Virtual Fields Method and a thermo-mechanical heterogeneous test carried out on a Gleeble 3500 system. To assess the feasibility of this novel calibration methodology, a modified version of the Johnson-Cook (J-C) model and dual-phase steel DP980 are selected. The three terms of the model that correspond to strain-hardening, temperature, and strain rate effects are calibrated simultaneously for the thermo-mechanical behaviour of this material.The calibration is attempted using a single test carried out at a constant displacement rate. This procedure is repeated for three tests at nominal strain rates of $10^{-4}, 10^{-3}$ and $10^{-2} \mathrm{~s}^{-1}$. Accurate predictions of the flow stress are attained, but the information of a single test is insufficient to capture the positive strain rate sensitivity of the material. The three tests are then combined in an experimental database to calibrate the model. The results show that the positive strain rate sensitivity is reasonably predicted in the considered range of temperatures. These results unveil the potential of this methodology to simplify the calibration process of thermo-elasto-viscoplastic constitutive model.
\end{abstract}

Keywords: Calibration, Virtual Fields Method, Heterogenous thermo-mechanical test, Full-field measurements, Thermo-elasto-viscoplasticity

\section{Introduction}

Temperature and strain rate have a significant influence on the flow stress of sheet metals. A precise description of their role is essential for the numerical simulation of heated assisted manufacturing processes, e.g. the simulation of hot and warm forming processes [1, 2, 3]. Furthermore, in standard manufacturing 5 processes, new generations of high strength steels reach high temperatures due to the heat generated by plastic deformation [4, which makes it essential to model accurately the effects of temperature and strain rate on this type of materials [5, 6].

Phenomenological thermo-elasto-viscoplastic constitutive models can represent the thermo-mechanical behaviour of metals and different formulations exist, depending on material behaviour, the range of strain, strain rate and temperature, as well as the computational cost [6, 7]. Generally speaking, phenomenological thermo-elasto-viscoplastic models are strongly non-linear and involve many material parameters that need to be calibrated. Classical calibration procedures at low to medium strain rates usually rely on isothermal tensile tests performed at different strain rates and temperatures [8, 9, 10. Other tests can be used, such as strain rate jump tests [6, 11, torsion [12] and shear [13] tests, but these are less common. In these classical

\footnotetext{
${ }^{*}$ Corresponding author

Email addresses: joao.martins52@ua.pt (J.M.P. Martins), sandrine.thuillier@univ-ubs.fr (S. Thuillier), gilac@ua.pt (A. Andrade-Campos)
} 
calibration procedures, the data collected from the tests are analysed under the assumption of homogeneous strain in the gauge length, leading to a single stress-strain curve output from each test that corresponds to a given temperature and strain rate. To cover a vast number of temperatures and strain rates at least an equal number of tests is required, which leads to long experimental campaigns.

Therefore, there has been a remarkable effort to reduce the number of tests by replacing classic procedures with a combination of full-field measurement techniques, heterogeneous tests and inverse methods, which was recently christened as Material Testing 2.0 [14. Full-field measurement techniques, such as Digital Image Correlation (DIC) [15], give complete displacement/strain maps at the surface of a specimen during a test, which, combined with heterogeneous tests [16, 17, 18, can provide a set of spatial points under different strain states, strain levels and strain rates. This information can also be enriched with temperature 25 measurements from the same test, obtained with a thermographic camera, for example. Thus, the required experimental database for the calibration can be collected from a few heterogeneous tests. The methodology proposed by Pottier et al. [19] is an example of this combination. Kinematic and temperature full-field measurements are combined in the same methodology to determine the inelastic heat fraction during quasi-static tensile tests. Another example is the recent inverse method proposed by Charlès and Le Cam 20. The

so proposed inverse method relies on the reconstruction of the heat source field, computed from both kinematic and temperature measurements, to calibrate constitutive models.

Nevertheless, the complexity of the loading conditions and the heterogeneity entails inverse methods [21, 22. Currently, the Finite Element Model Updating (FEMU) and the Virtual Fields Method (VFM) are the most used [14. In the case of FEMU, a finite element (FE) model of the heterogeneous test is required and the 35 calibration of the constitutive models is carried out through an iterative procedure that searches for the best set of material parameters to minimise the difference between experimental and numerical data 23, 24]. Although this method is quite popular, it has a major drawback. Indeed, the large number of $\mathrm{FE}$ calculations which is usually required leads to a high computational cost. Alternatively, the VFM provides a more efficient solution in terms of computational cost [25, 26, 27. Derived from the principle of virtual work, this

40 inverse method conducts the calibration process by balancing internal and external work, quantities that are computed from the measured displacements field and load, respectively 25]. Since the pioneering work of Grédiac 28 to characterize material properties of composites materials, the VFM has been successfully applied and consequently, adapted to calibrate different types of constitutive models. Nowadays, it is an appealing solution for calibrating non-linear constitutive models in the fields of plasticity [29, 30, 31] and 45 viscoplasticy [32, 33, 34].

In the framework of low strain rate values, the work of Gramma et al. 32 aims at the simultaneous calibration of all the parameters of the Anand model with a single test. The authors analyse the sensitivity of the procedure to different loading ratios and rates in order to design a test with the necessary information. Jones et al. [33] investigated the calibration of the Bammann-Chiesa-Johnson viscoplastic model using a so heterogeneous test with a specimen geometry similar to a capital letter "D". The simultaneous calibration of the full set of parameters raised the problem of non-uniqueness of the solution. Nevertheless, the authors reached valid solutions, which were considered functionally equivalent to the loading conditions present in the test. Enrichment of the database was the solution suggested by the authors to mitigate the non-uniqueness problem. Valeri et al. 34 proposed a methodology based on the VFM to calibrate the thermo-viscoplastic 55 Johnson-Cook model. The strain-hardening term is calibrated following a classical procedure and then only the temperature and strain rate terms are calibrated using the VFM. The temperature, the averaged strain and strain rate obtained from isothermal uniaxial tests composed the experimental database. The authors reached accurate descriptions of the flow stress from room temperature up to $900{ }^{\circ} \mathrm{C}$. Although less effective in computational terms, FEMU-based approaches relying on full-field kinematic and thermal data have been so proposed in recent years. Rose and Menzel [35] proposed a staggered identification methodology, which considered three calibration phases dedicated to elastic, plastic and thermal material parameters. Data from tensile tests carried out at room temperature were used. Archer et al. [36 also proposed a FEMU-based approach to calibrate creep parameters of an environmental barrier coating. The authors adopted a staggered approach to calibrate all the parameters of the finite element model and full-field data of a devised 65 test representative of turbine engine environments.

The aim of the present work is to take a further step towards improving and simplifying the calibra- 
tion procedure of thermo-mechanical constitutive models. The VFM is combined with a heterogeneous thermo-mechanical test to fully calibrate a set of constitutive parameters. Thus, taking advantage of the computational efficiency of this inverse method and the richness of data present on heterogeneous tests.

70 The heterogeneous test used in this work is carried out on a Gleeble thermo-mechanical simulator. At this early stage, the objective lies more in the proof of concept regarding the combination of the VFM and the thermomechanical test than on reaching bulletproof identifications.

After this introduction section, the experimental programme is presented in section 2. The Gleeble system and the conditions of the tests are introduced, as well as the data acquisition equipment. Section 3 contains

75 the description of the adopted constitutive model, the VFM calibration procedure and the finite element model used to assess the validity of the results. Section 4 is dedicated to the results. First, the experimental data are presented and then the calibration results. The calibration process is analysed for an experimental database composed of a single test and then for an experimental database composed of the three tests. In section 5, concluding remarks close the article.

\section{Experimental programme}

In this study, a heterogeneous thermo-mechanical test performed on Gleeble 3500 thermo-mechanical system is used to create an experimental database for the calibration process. This test has an overlap of heterogeneous temperature and strain fields, which can provide information on the mechanical behaviour of

the material for a considerable range of temperatures and strain rates.

\subsection{Gleeble system}



Figure 1: Gleeble 3500 thermo-mechanical system coupled with DIC system (GOM-Aramis) and a FLIR thermographic camera. The Gleeble chamber with specimen prepared for testing and illuminated by polarized blue light is shown in the image below. The three thermocouples used to measure the temperature along the specimen are labelled. 
Fig. 1 illustrates the experimental setup that includes the Gleeble 3500 thermo-mechanical used in this study. This system combines a hydraulic servo system able to impose tension or compression forces and a direct resistance heating system [37. A temperature value can be imposed at the centre of the specimen and held constant during the mechanical test, using the direct resistance heating system. The heating process is controlled by a thermocouple signal that provides an accurate temperature measurement. However, a temperature gradient is developed from the centre to the extremities of the specimen due to the watercooled jaws carriers. Frequently deemed as adverse [38, 39, 40, this gradient is an asset for the present study since it triggers a heterogeneous deformation process.

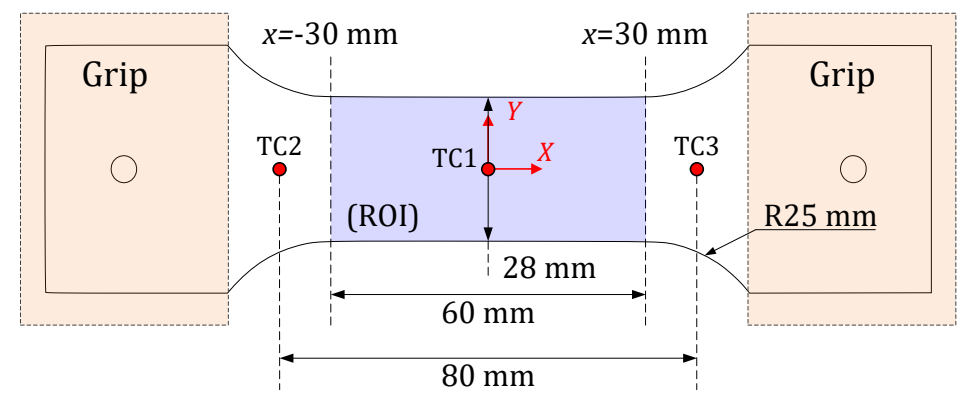

Figure 2: Tensile specimen geometry with the region of interest (ROI) represented in blue and the position of the thermocouples, TC1, TC2 and TC3, represented by the red circles.

\subsection{Material and specimen geometry}

A dual-phase steel DP980 is considered for this study [41, 42. Fig. 2 presents the tensile specimen geometry. The specimens are cut from a rolled sheet with $1.75 \mathrm{~mm}$ thickness. The longitudinal direction (x-direction in Fig. 2) of the specimens is aligned with the rolling direction of the sheet.

\subsection{Experimental procedure and data acquisition}

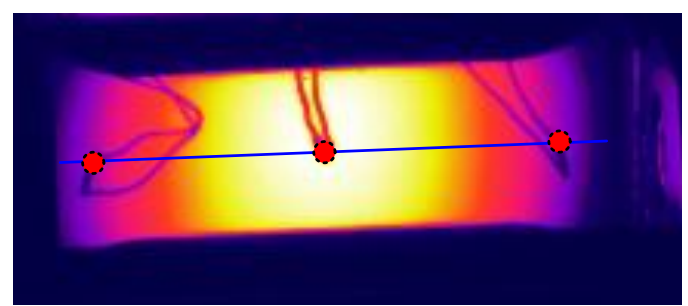

Figure 3: Temperature field of the test V2 recorded by the FLIR thermographic camera. The highest temperatures are shown in yellow and the lowest in violet. The line passing through the mid-plane of the specimen $(y=0$ mm) is represented in blue and the position of the thermocouples by the red circles.

The specimens are loaded under a constant displacement rate. Three displacement rates evenly distributed in the logarithmic scale are selected to conduct the tensile tests: $0.006,0.06$ and $0.6 \mathrm{~mm} \mathrm{~s}^{-1}$. The tests corresponding to this displacement rates will be called V1,V2 and V3, for the sake of simplicity. According to the length of the region with constant cross-section $(60 \mathrm{~mm})$, these displacement rates correspond to nominal strain rates of $10^{-4}, 10^{-3}$ and $10^{-2} \mathrm{~s}^{-1}$, respectively. Regarding the thermal field, a temperature value of $500{ }^{\circ} \mathrm{C}$ is the imposed on the centre of the specimens. The deformation process starts after a heating phase to ensure that the reference value of $500^{\circ} \mathrm{C}$ is reached at the centre of the specimen. 
The local kinematic measurements are acquired through the DIC system GOM-Aramis 43. The adopted DIC settings for this system are summarized in Table 1. The random black-and-white pattern was applied with commercial paints provided by Jelt (0057 and 5641 Colorjelt). At high temperatures $\left(>700{ }^{\circ} \mathrm{C}\right)$, the radiation emitted by the heated specimen can reduce the accuracy of the DIC measurements and it is common practise to use blue light illumination combined with filters in front of the cameras 34, 44, 45. Although in this study the temperature range goes up to $500{ }^{\circ} \mathrm{C}$, it is also used a polarised blue light combined with blue pass filters (IF 039 supplied by schneider kreuznach) to avoid potential errors from the emitted radiation. The temperature field is monitored through three thermocouples (TC1, TC2 and TC3), which are welded in the specimens, and through a FLIR thermographic camera X6580SC. The latter equipment provides the spatial distribution of temperature across the entire surface of the specimens. The three thermocouples (see Fig. 2 and 3) are used as references for the coordinate system, as well as to determine the emissivity of the surface.

The temperature measurements of the three thermocouples (TC1, TC2 and TC3) for each test are shown

\begin{tabular}{ll}
\hline Camera & DALSA FALCON 4M60 \\
Sensor & $2352 \times 1728 \mathrm{px}^{2}$ \\
Lens & schneider kreuznach $35 \mathrm{~mm}$ \\
Aperture & $\mathrm{f} / 8$ \\
Field of view & $90 \times 35 \mathrm{~mm}^{2}$ \\
Image scale & $12 \mathrm{px} / \mathrm{mm}$ \\
Stereo-angle & $25^{\circ}$ \\
Stand-off distance & $440 \mathrm{~mm}$ \\
Patterning technique & White spray (base coat) \\
& with black speckles \\
Pattern feature size & $0.25 \mathrm{~mm} / 3$ px \\
DIC software & ARAMIS $3 \mathrm{D}$ 4M \\
& version $6.3 .1-2$ \\
Technique used & Stereo Digital Image Correlation \\
Subset size & $20 \times 20 \mathrm{px}^{2}$ \\
Step size & $12 \mathrm{px}$ \\
Subset shape function & linear \\
Matching criterion & Sum of square differences (SSD) \\
Strain window & $104 \times 58 \mathrm{px}^{2}$ \\
\hline
\end{tabular}

Table 1: DIC settings.

in Fig. 4. The displacement $\left(\mathrm{u}_{x}\right)$ in the $x$-axis is calculated as the difference of the average displacement value at the boundaries $\mathrm{x}=-30 \mathrm{~mm}$ and $\mathrm{x}=30 \mathrm{~mm}$ of the region of interest (ROI) (see Fig. 2). As can be seen, the temperature remains nearly the same throughout each test, which results from the Gleeble's very precise control system, which preserves the temperature at the TC1 thermocouple, and from the high thermal conductivity of the material. Moreover, the temperature varies mainly along the longitudinal direction of the specimen (x-direction), while the high conductivity of the steel leads to meaningless differences along the transversal direction (y-direction), less than $2 \%$. Therefore, the variation along the transversal direction is neglected in the analysis of the tests, as well as in the calibration procedure.

Fig. 5 shows the spatial distribution of temperature for the beginning of each test along a line passing through the mid-plane of the specimens $(y=0 \mathrm{~mm})$, as shown in Fig. 3. The red squares represent the thermocouples measurements, and the colour dots represent the thermal camera measurements. For the positions of the thermocouples, the measurements of the thermographic camera show abrupt temperature drops. This is caused by the different emissivity of the thermocouples when compared with the surface of the specimens. For the three cases, the temperature field reaches its maximum value at the centre of the specimens and presents a bell-shaped distribution that is characteristic of this system [37, 38, 40. From the 


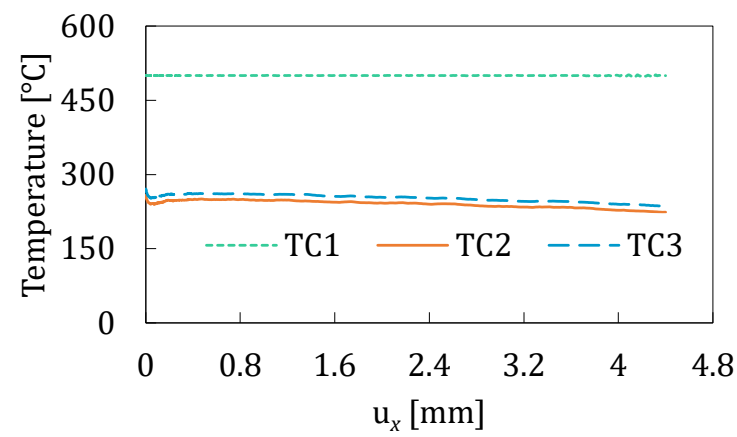

(a) V1



(b) V2

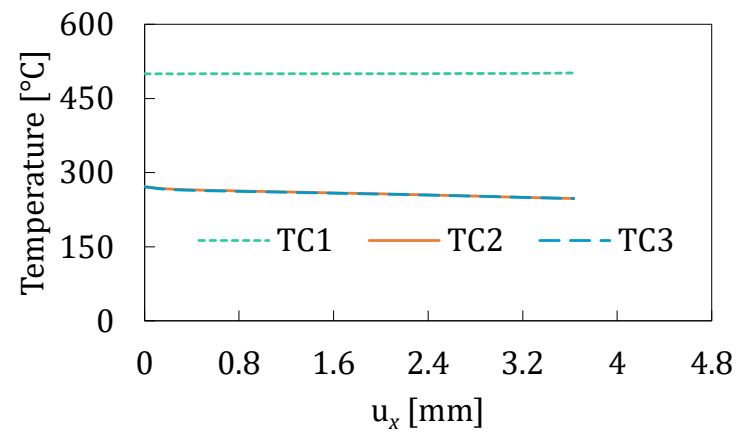

(c) V3

Figure 4: Temperature evolution measured by the three thermocouples TC1, TC2 and TC3 for the three tests: (a) V1, (b) V2 and (c) V3. The temperature is almost constant during the deformation process, which results from Gleeble's very precise control system and the high thermal conductivity of the material.

centre to the extremities of the specimens, it is observed the decrease of temperature that leads to values around $360^{\circ} \mathrm{C}$ at the boundaries of the ROI $(x=-30$ and $x=30 \mathrm{~mm})$, i.e. a temperature gradient of $140^{\circ} \mathrm{C}$. This temperature distribution can be described by a $2^{\text {nd }}$ order polynomial [46, 47], as can be seen in Fig. 5. The squared values of the correlation coefficient $\left(\mathrm{R}^{2}\right)$ are around 1.0, which proves the very good agreement of the $2^{\text {nd }}$ order polynomial with the experimental measurements.

A stress analysis for the central region of the specimen is presented in the results section. For this analysis, it is assumed a uniaxial stress state with homogeneous deformation for the central section of the specimens in between $x=-2$ and $x=2 \mathrm{~mm}$. Based on that, the Cauchy stress $(\sigma)$ is computed from the measured load and considering the actual cross-sectional area of the specimen. The actual cross-sectional area is computed assuming volume conservation. In this case, $\varepsilon_{x x}^{\text {avg }}$ is the average logarithmic strain component for that central region.

The strain rate field can be considered as another source of information for the calibration, hence it is also analysed in the results section. Strain rate values $\left(\dot{\varepsilon}_{x x}\right)$ are computed from the $\varepsilon_{x x}$ logarithmic strain component through forward finite-differences.

\section{Theory}

\subsection{Constitutive model}

The original version of Johnson-Cook model [12] is widely adopted to represent the response of metals under a large range of temperatures and strain rates. However, this original formulation of the J-C model 


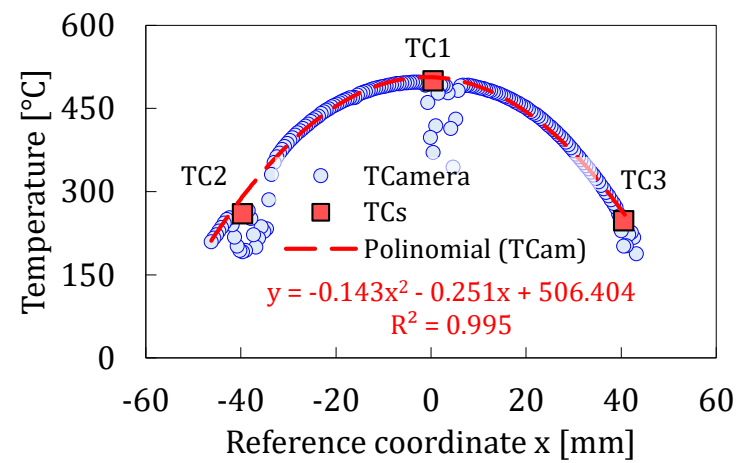

(a) V1

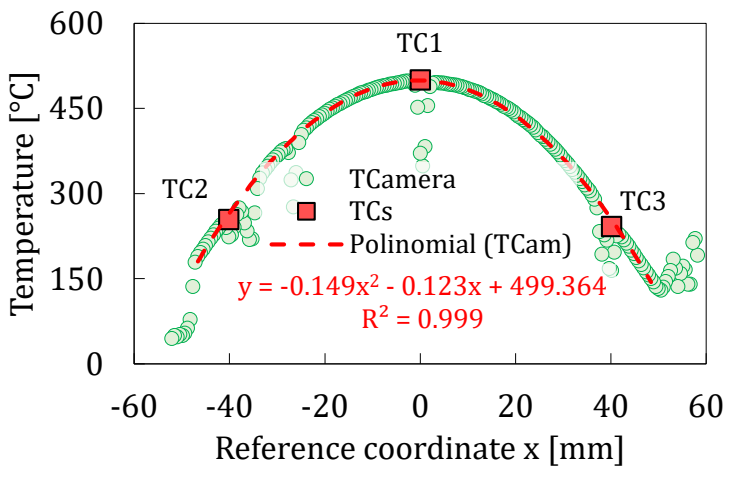

(b) V2

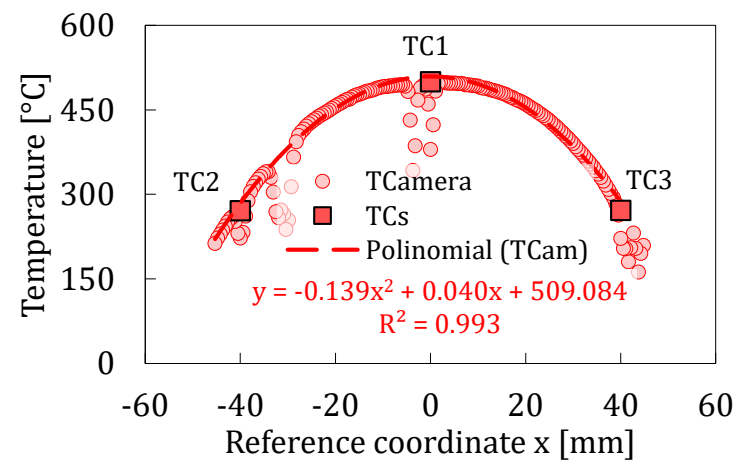

(c) V3

Figure 5: Spatial temperature distribution for each test along a line passing through the mid-plane of the specimen $(y=0 \mathrm{~mm})$ (see Fig. 3): (a) V1, (b) V2 and (c) V3. The temperature distribution of the three tests is described by a $2^{\text {nd }}$ order polynomial function. The correlation coefficient $\left(\mathrm{R}^{2}\right)$ has values around 1.0 which proves the good agreement between the $2^{\text {nd }}$ order polynomial function and the experimental measures.

lacks flexibility and several modifications have been proposed [48. For example, regarding the strainhardening term, the original power model has been replaced in several works by a combined Swift-Voce model providing superior accuracy in the description of the mechanical behaviour of dual-phase steels [6, 49, 5]. Thus, a modified version of the J-C model is selected for this work. This modified version assumes the following form

$$
\sigma_{y}=H\left(\bar{\varepsilon}^{\mathrm{p}}\right) \cdot G(T) \cdot F\left(\dot{\bar{\varepsilon}}^{\mathrm{p}}\right)
$$

where,

$$
\begin{gathered}
H\left(\bar{\varepsilon}^{\mathrm{p}}\right)=\alpha \cdot\left[K \cdot\left(\varepsilon_{0}+\bar{\varepsilon}^{\mathrm{p}}\right)^{n}\right]+(1-\alpha) \cdot\left[\sigma_{0}+\left(\sigma_{\mathrm{sat}}-\sigma_{0}\right) \cdot\left(1-\exp \left(-C_{y} \cdot \bar{\varepsilon}^{\mathrm{p}}\right)\right)\right], \\
G(T)=\left[1-\left(\frac{T-T_{\mathrm{tr}}}{T_{\mathrm{m}}-T_{\mathrm{tr}}}\right)^{m}\right],
\end{gathered}
$$

and

$$
F\left(\dot{\bar{\varepsilon}}^{\mathrm{p}}\right)=\left[1+C \ln \left(\frac{\dot{\bar{\varepsilon}}^{\mathrm{p}}}{\dot{\varepsilon}_{0}}\right)\right] .
$$

The flow stress $\left(\sigma_{y}\right)$ is composed by three functions, $H\left(\bar{\varepsilon}^{\mathrm{p}}\right), G(T)$ and $F\left(\dot{\bar{\varepsilon}}^{\mathrm{p}}\right)$, which represent, respectively, the strain-hardening, temperature and strain rate effects. These three terms are governed by the variables: equivalent plastic strain $\bar{\varepsilon}^{\mathrm{p}}$, temperature $T$ and equivalent plastic strain rate $\dot{\bar{\varepsilon}}^{\mathrm{p}}$. The strain-hardening term 
contains seven material parameters, $\alpha, K, n, \sigma_{0}, \sigma_{\text {sat }}$ and $C_{y}$ and $\varepsilon_{0}=\left(\sigma_{0} / K\right)^{1 / n}$. The term $G(T)$ has three parameters $T_{\mathrm{tr}}, T_{\mathrm{m}}$ and $m$, and it is only active when the actual temperature $T$ is higher than the transition temperature $T_{\mathrm{tr}}$. The term $F\left(\dot{\bar{\varepsilon}}^{\mathrm{p}}\right)$ has two parameters $C$ and $\dot{\varepsilon}_{0}$, and for this term to be active, $\dot{\bar{\varepsilon}}^{\mathrm{p}}$ must be higher than $\dot{\varepsilon}_{0}$.

The formulation of the J-C model is usually classified as a decoupled formulation because the strainhardening, temperature and strain rate effects are modelled as independent phenomena [50, each one in a single term. Moreover, the J-C model does not consider temperature or strain rate history effects. This type of formulation has the advantage of easy implementation.

A common approach to calibrate the $\mathrm{J}-\mathrm{C}$ model is to divide the process into three steps, each step corresponding to the individual calibration of a term. In this work, the simultaneous calibration of the three terms is analysed. Some parameters are assumed to be known a priori, such as $T_{\mathrm{tr}}=25{ }^{\circ} \mathrm{C}$ and $T_{\mathrm{m}}=1000{ }^{\circ} \mathrm{C}$. The value assumed for $T_{\mathrm{m}}$ is a common value for DP980 [5]. In the case of $T_{\mathrm{tr}}$, it is intended to reach a model calibration for a large range of temperatures. The parameter $\dot{\varepsilon}_{0}$ is also fixed to the value of $1.0 \cdot 10^{-5} \mathrm{~s}^{-1}$. Such a low value is defined because the positive effect of strain rate occurs very early in the deformation process of DP980, which implies the activation of the strain rate term for low values of the equivalent plastic strain rate. Moreover, assuming this parameter as known also mitigates the problem of multiple solutions [51]. The value of $\alpha$ is defined to be equal to 0.5. Note that setting $\alpha$ as a parameter to be identified is useless because $K$ also weights the hardening terms.

Moreover, the model is developed within the framework of associated flow rule. The von Mises yield criterion and isotropic linear elasticity described by the generalized Hooke's law are adopted. The identification of the elastic parameters is not part of this work, thus based on the literature the value of 0.3 is assumed for the Poisson's ratio [1]. Concerning Young's modulus, a dependence on the temperature is observed, which is according to the data reported in the literature for dual-phase steels [52, 53. For the DP980 used in this study, average Young's modulus values of $185 \mathrm{GPa}$ and $106 \mathrm{GPa}$ are determined from stress-strain curves at room temperature and $500{ }^{\circ} \mathrm{C}$, respectively. As no other values are available in this range of temperatures, it is adopted a bilinear-piecewise equation to model the dependence of Young's modulus on temperature. This bilinear-piecewise equation is a simplified approach that takes into account a steepest decrease of Young's modulus with temperature.

$$
E(T)=\left\{\begin{array}{lll}
185 & \text { if } & T \leq 492{ }^{\circ} \mathrm{C} \\
-9.93 \cdot T+5074 & \text { if } & 492{ }^{\circ} \mathrm{C}<T<500{ }^{\circ} \mathrm{C} \quad[\mathrm{GPa}] . \\
106 & \text { if } & T \geq 500{ }^{\circ} \mathrm{C}
\end{array}\right.
$$

Note that this bilinear-piecewise equation is optimised to reach the best fit at the beginning of the load curves of the heterogeneous tests.

\subsection{Virtual Fields Method}

The VFM calibration process relies on the principle of virtual work (PVW) written for a suitable set of virtual fields. The PVW is combined with full-field kinematic measurements acquired during a mechanical test and the measured load obtained from a load-cell. The calibration is performed through the enforcement of the equilibrium condition between the external and internal virtual work. In the framework of finite strain theory, assuming body forces as null and quasi-static conditions, the PVW can be written in the reference configuration as follows

$$
\underbrace{\int_{\Omega_{0}} \mathbf{P}: \operatorname{grad} \mathbf{U}^{*} \mathrm{~d} V}_{W_{\text {int }}}-\underbrace{\int_{\Gamma_{0}^{f}} \overline{\mathbf{T}} \cdot \mathbf{U}^{*} \mathrm{~d} S}_{W_{\mathrm{ext}}}=0,
$$

where $\mathbf{P}$ is the first Piola-Kirchhoff stress tensor, $\mathbf{U}^{*}$ is the virtual displacement vector expressed in the reference configuration $\left(\mathbf{U}^{*}=\mathbf{U}^{*}(\mathbf{X})\right.$ ), as well as grad $\mathbf{U}^{*} . \overline{\mathbf{T}}$ is the first Piola-Kirchhoff stress vector prescribed on the boundary $\Gamma_{0}^{f}$ with the reference surface area $S$. Similarly, the first integral of Eq. 6 is written over the reference volume $V$. The virtual displacement field $\left(\mathbf{U}^{*}\right)$ must be kinematically admissible, 
continuous and differentiable [25, 54].

The first Piola-Kirchhoff stress tensor $\mathbf{P}$ is obtained through the Piola transformation as

$$
\mathbf{P}=\operatorname{det}(\mathbf{F}) \boldsymbol{\sigma} \mathbf{F}^{-\mathrm{T}},
$$

where $\operatorname{det}(\mathbf{F})$ is the determinant of the deformation gradient $\mathbf{F}$ and $\mathbf{F}^{-\mathrm{T}}$ is the transpose of its inverse. The Cauchy stress tensor $\boldsymbol{\sigma}$ is computed from the strain tensor $\varepsilon$, which is determined through the kinematic full-field measurements, and by means of a previously selected constitutive model combined with an initial set of material parameters $\boldsymbol{\xi}$. In the present case, the assumed thermo-elasto-viscoplastic constitutive model (Eq. 1) takes into account temperature and strain rate effects, which brings two more variables into the problem, time $t$ and temperature $T$. Therefore,

$$
\boldsymbol{\sigma}=\boldsymbol{\sigma}(\varepsilon, \boldsymbol{\xi}, t, T) .
$$

The strain tensor is computed from the measured displacement fields $\mathbf{u}$ by means of the deformation gradient F. Using the theorem of polar decomposition, the deformation gradient can be decomposed as

$$
\mathbf{F}=\mathbf{V R},
$$

where $\mathbf{V}$ is the left Cauchy stretch tensor and $\mathbf{R}$ is the orthogonal rotation tensor. The strain field can be computed for each time instant as the Hencky strain tensor

$$
\varepsilon=\ln \mathbf{V} .
$$

Moreover, the computation of the Cauchy stress tensor is conventionally expressed in a local material frame free of rigid body rotations. Thus, the strain tensor in this local co-rotational frame can be computed as

$$
\hat{\varepsilon}=\mathbf{R}^{\mathrm{T}} \varepsilon \mathbf{R} .
$$

Full-field measurements are usually obtained as a discrete number of material points in a ROI belonging to the surface of the specimen. The computation of kinematic quantities can then be carried out using, for example, shape functions for discrete points in the ROI. The stress tensor and the virtual fields are also computed for the same locations and become representative of the average quantities of small regions. Moreover, as the through thickness information is not usually available, it is assumed plane stress condition. The thickness estimation, which is required for the Piola transformation, is computed through the Poisson's ratio and the assumption of incompressible plasticity. For further details on the implementation of these assumptions, the reader is referred to [55. Furthermore, a recent study by Jones et al. [56] shows that the assumptions of incompressible plasticity and plane stress are valid options in VFM.

Based on this discrete form of the data, the first integral (Eq. 6), which corresponds to the internal virtual work, is approximated by a discrete sum as follows

$$
W_{\text {int }}(\boldsymbol{\xi})=\sum_{i=1}^{n_{\mathrm{e}}} \mathbf{P}\left(\varepsilon_{i}, \boldsymbol{\xi}, t, T_{i}\right): \operatorname{grad} \mathbf{U}_{i}^{*} A_{i} e_{i},
$$

where $n_{\mathrm{e}}$ represents the number of points. $A_{i}$ and $e_{i}$ represent the area and the thickness for the location of these points. Note that adopting the reference configuration has the advantage that the area $A_{i}$ and thickness $e_{i}$ do not need to be updated as the deformation process evolves. The same happens with the computation of the virtual fields that is only performed for the reference configuration.

The external virtual work is computed from the load acquired during the mechanical test. This computation can be simplified through a proper choice of the virtual fields. In order to have this simplification, the selected virtual fields must be constant on the boundary $\Gamma_{0}^{f}$, leading to the computation of the second integral in 
Eq. 6 as follows

$$
W_{\text {ext }}=\mathbf{U}^{*} \cdot \int_{\Gamma_{0}^{f}} \overline{\mathbf{T}} \mathrm{d} S=\mathbf{U}^{*} \cdot \mathbf{F}^{\mathrm{load}},
$$

where $\mathbf{F}^{\text {load }}$ is the resultant of the force acting on the boundary. This simplification is a major asset of the VFM because the distribution of the force vector on the boundary is usually unknown.

\subsubsection{Objective function}

The PVW can be written for different time steps and for different virtual fields in order to enrich the objective function. Relevant information from the history of the mechanical test and from different points in the spatial domain of the ROI can thus be explored. Hence, the objective function for the VFM can be written in a least-square framework as

$$
\varphi(\boldsymbol{\xi})=\sum_{k=1}^{n_{\mathrm{vf}}} \frac{1}{n_{\mathrm{vf}}}\left\{\sum_{j=1}^{n_{\mathrm{t}}} \frac{1}{n_{\mathrm{t}}}\left[\sum_{i=1}^{n_{\mathrm{e}}} \mathbf{P}\left(\boldsymbol{\varepsilon}_{i j}, \boldsymbol{\xi}, t_{j}, T_{i j}\right): \operatorname{grad} \mathbf{U}_{i k}^{*} A_{i} e_{i j}-\mathbf{U}_{k}^{*} \cdot \mathbf{F}_{j}^{\text {load }}\right]\right\}^{2},
$$

where $n_{\mathrm{vf}}$ is the number of virtual fields selected and $n_{\mathrm{t}}$ the number of time steps considered. The calibration of the constitutive model is performed through the minimisation of the cost function given by Eq. 14. The material parameters $\boldsymbol{\xi}$ are found when the computed stress field minimises the difference between $W_{\text {int }}$ and $W_{\text {ext }}$ for all time steps and virtual fields. Moreover, several tests can be used, and in that case, the objective

\subsubsection{Virtual fields selection and identification procedure}

One of the key elements of VFM is the selected set of virtual fields. The virtual fields can be seen as weights attributed to the stress field, which may vary in space and even in time, and are independent of the measured displacement fields. These must be kinematically admissible and can be any continuous and differentiable function. Moreover, in order to have a simplified computation of the external virtual work, these should also be constant on the boundaries where forces are applied. For the calibration of nonlinear constitutive models, there are three approaches to select a suitable set of virtual fields. Two of these approaches are automatic strategies and require a low level of user intervention [55, 57. The other approach, called manually defined virtual fields, depends exclusively on the user intervention, since it is the user who must develop the appropriate set according to the boundary conditions of the test. Compared to the other strategies, manually defined virtual fields strategy has the main advantages of low computational cost and ease of implementation. For these reasons, this is the strategy adopted in the present work.

Recently, it has been confirmed that the number of virtual fields plays an important role in the accuracy of the VFM [31, 58. Increase the number of virtual fields in Eq. 14 is just a way to improve the objective function with more information contained in the stress fields. A total of 9 virtual fields are selected for this 
work. Note that $n_{\mathrm{vf}}>\operatorname{dim}(\boldsymbol{\xi})$. The selected set is defined as follows

$$
\begin{array}{ll}
\mathbf{U}_{1}^{*}= \begin{cases}U_{X}^{*}=\frac{X}{L} \\
U_{Y}^{*}=0\end{cases} & \mathbf{U}_{2}^{*}=\left\{\begin{array}{l}
U_{X}^{*}=0 \\
U_{Y}^{*}=\cos \frac{\pi X}{2 L}
\end{array}\right. \\
\mathbf{U}_{3}^{*}=\left\{\begin{array}{l}
U_{X}^{*}=0 \\
U_{Y}^{*}=\frac{Y}{W} \cos \frac{\pi X}{2 L}
\end{array}\right. & \mathbf{U}_{4}^{*}=\left\{\begin{array}{l}
U_{X}^{*}=\sin \frac{\pi X}{L} \sin \frac{\pi Y}{L} \\
U_{Y}^{*}=\sin \frac{\pi X}{L} \sin \frac{\pi Y}{L}
\end{array}\right. \\
\mathbf{U}_{5}^{*}=\left\{\begin{array}{l}
U_{X}^{*}=0 \\
U_{Y}^{*}=\sin \frac{\pi X}{L} \sin \frac{\pi Y}{L}
\end{array}\right. & \mathbf{U}_{6}^{*}=\left\{\begin{array}{l}
U_{X}^{*}=0 \\
U_{Y}^{*}=\frac{Y(|X|-L)}{W L}
\end{array}\right. \\
\mathbf{U}_{7}^{*}=\left\{\begin{array}{l}
U_{X}^{*}=\frac{Y\left(X^{2}-L^{2}\right)}{L^{2} W} \\
U_{Y}^{*}=0
\end{array}\right. & \mathbf{U}_{8}^{*}=\left\{\begin{array}{l}
U_{X}^{*}=0 \\
U_{Y}^{*}=\frac{Y^{2}}{W^{2}} \sin \frac{\pi X}{L}
\end{array}\right. \\
\mathbf{U}_{9}^{*}=\left\{\begin{array}{l}
U_{X}^{*}=\left[\exp \left(\frac{H^{2}-X^{2}}{H^{2}}\right)-1\right] \sin \frac{\pi X}{L} \\
U_{Y}^{*}=0
\end{array}\right. &
\end{array}
$$

The constants $L$ and $W$ represent half the length and width of the ROI, respectively. $X$ and $Y$ are the coordinates in the reference frame. Note that the reference frame is located at the specimen centre as shown in Fig. 2.

In the case of static equilibrium, the first virtual field is the only one that gives a non-zero value in the computation of the internal virtual work. The component $\partial U_{X}^{*} / \partial X$ of the gradient is the only component with non-zero value for this virtual field and it has constant distribution in ROI $\left(\partial U_{X}^{*} / \partial X=1 / L\right)$. In this case, the internal virtual work is balanced with the external virtual work computed from the measured load. The other virtual fields have non-constant spatial distributions, which give a non-constant distribution of weight between normal and shear components in the computation of the internal virtual work.

Another key element of the VFM is the reconstruction of the stress field. In this study, a stress integration algorithm based on a backward-Euler scheme combined with a predictor/corrector method is adopted. Examples of this kind of algorithms can be found in [59, 60]. This type of algorithms is also generally adopted in FE analysis and remains the most widely used type in the reconstruction of the stress field for inverse identification methods 51, 55, 56, 61. These are known for satisfying the yield condition after an iterative process and to be unconditionally stable. The downside of these algorithms is the required computation of the second derivative of the yield function (associate flow rule) or plastic potential (nonassociated flow rule). Moreover, when compared with new methods specially developed to handle full-field data 62, 63, which can be called direct methods since no iteration process is required, the computational cost is higher. For further details on this topic, see for instance [51, 55, 56, 61, 62, 63.

An in-house VFM code developed using Fortran programming language is used in this study. A gradientbased Levenberg-Marquardt optimisation method 64 is selected to solve the optimisation process. The required Jacobian matrix for the Levenberg-Marquardt method is calculated by forward finite differences. The convergence criteria for this method are established as: the objective function becomes lower than a tolerance or the relative difference between parameters in consecutive iterations is lower than or equal to $1.0 \cdot 10^{-10}$.

The VFM procedure is depicted in Fig. 6. The iteration cycle is delimited by the red area, which includes the computation of IVW and EVW for each new set of parameters, the evaluation of the objective function and the optimisation process. The input data for the VFM code are the full-field displacements, from which the strain field is deduced.

In the present work, the raw full-field displacements are post-treated into Matlab 65] before using as input data. The displacement fields corresponding to each time step are interpolated onto a rectangular grid covering the entire area of the ROI. For the specific test in hands, the grid is divided into regular elements with an area of $1 \mathrm{~mm}^{2}$, which makes a total of 1769 data points for the spatial domain corresponding the nodes of the grid. After interpolation, the displacements are smoothed through space and time. The spatial smoothing is performed using the moving average method with a span of 15 and the temporal 


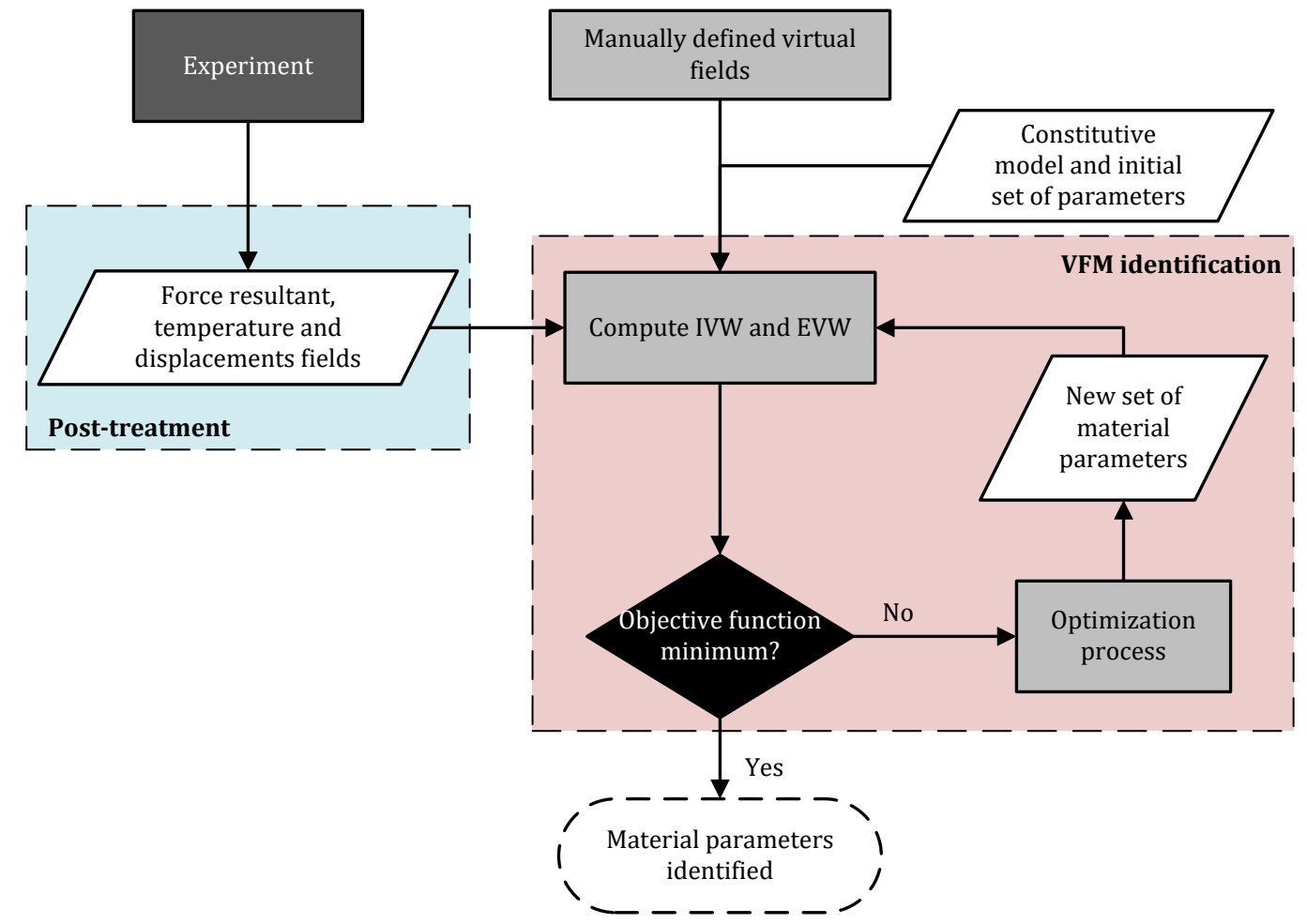

Figure 6: Flowchart representing the algorithm for the identification procedure of VFM. This procedure starts with defining of the set of virtual fields, which in the present work are manually defined. Follows the definition of constitutive model and initial set of parameters for this model. After post-treatment, the force resultant, temperature and displacement fields are the input data to compute the IVW and EVW. An optimisation method leads the minimisation of the objective function.

smoothing is performed using the Savitzky-Golay method with a polynomial order of 3 and frame length of 21. After completing this procedure, a reduced number of time steps, up to the maximum load value, is selected and become the input data for VFM computations. The reduced number of time steps decreases the computational time and increases the size of the strain increments, which mitigates the impact of strain noise on the computation of the stress field. The strain tensor is computed for the centroid of the elements using bilinear shape functions and the stress tensor is also computed for the same location. Thus, the number of data points $\left(n_{\mathrm{e}}\right)$ in Eq. 14 is 1680 .

The temperature field is also an input for the reconstruction of the stress field. The polynomial functions presented in Fig. 5 are used to impose the measured temperature values in each element of the grid. Note that only the gradient along the longitudinal direction (x-direction) of the specimen is considered. Moreover, as shown in Fig. 4, the temperature for the positions of the thermocouples is almost constant during the tests. Therefore, it is assumed that the elements of the grid hold the same temperature value for all the selected time steps.

\subsection{Finite element model}

Finite element simulations of the 3 tests are performed to validate the calibration results. Therefore, a 3D FE model of the ROI of the tensile specimen (Fig. 22 is built in the Abaqus ${ }^{\circledR}$ standard. This model was previously presented in 58. Symmetry boundary conditions are assumed, and only half of the thickness of the specimen is represented. An eight-node solid element with reduced integration (C3D8R) is adopted for the mesh, which is composed of $60 \times 28 \times 3$ regular elements. Therefore, the top surface of the model is divided by $60 \times 28$ elements, which corresponds to $1 \mathrm{~mm}^{2}$ subregions. These dimensions are in the same order of magnitude as the selected subset size for the DIC results. The model is coupled to the UMAT subroutine 
with the modified J-C model presented in section 3.1. The simulations of the tests are performed for the respective loading conditions, which consist of different nominal strain rates: $10^{-4}, 10^{-3}$ and $10^{-2} s^{-1}$. The

boundary conditions are the displacements imposed on the boundaries $x=-30$ and $x=30 \mathrm{~mm}$, which were extracted from the DIC measurements of each test. The top surface of the FE model is presented in Fig. 7. The temperature distributions presented in Fig. 5 are imposed on the respective test by means of the $2^{\text {nd }}$ order polynomial functions.

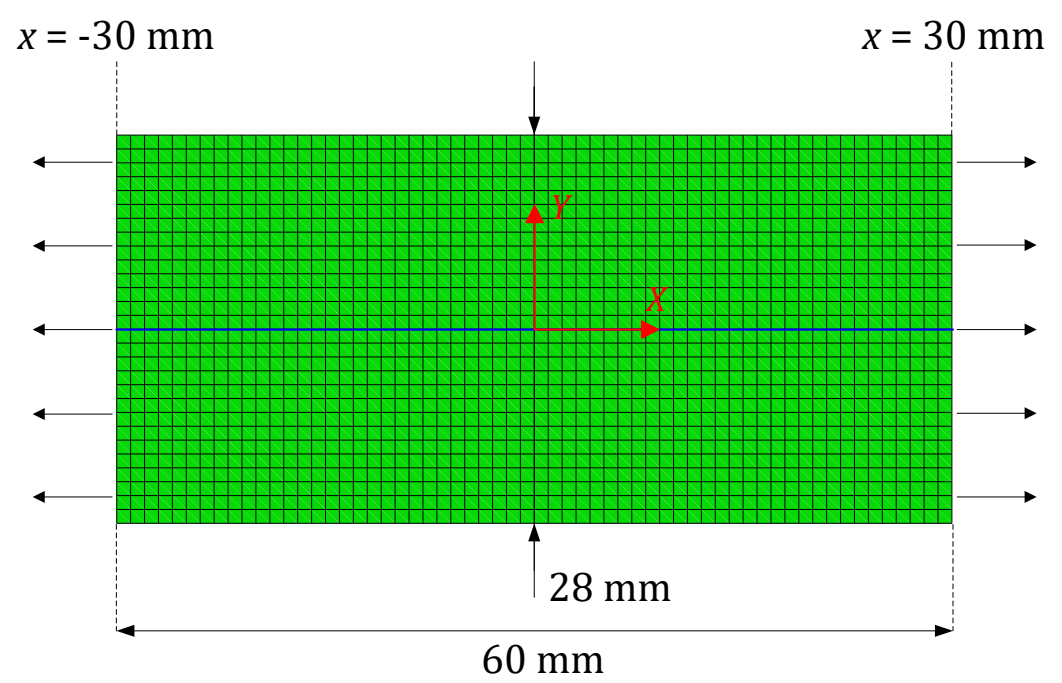

Figure 7: FE model representing the ROI of the tensile specimen (see Fig 2). The figure shows only the top surface, with a mesh of $60 \times 28$ elements. Each element corresponds to $1 \mathrm{~mm}^{2}$ subregion on the top surface of the model. The displacement are imposed on the boundary $x=-30$ and $x=30 \mathrm{~mm}$. The blue line passes through the mid-plane of the specimen $(y=0 \mathrm{~mm})$.

\section{Results}

\subsection{Experimental results}

\subsubsection{Load-displacement and stress-strain curves}

Fig. 8 presents the load-displacement curves up to rupture for the three displacement rates. Note that, although only a single curve is presented for each displacement rate, repeatability is observed for the three cases. The material shows a positive strain rate sensitivity. The maximum load value indicated by the black circles corresponds to $22.3,27.0$ and $30.5 \mathrm{kN}$, for the tests V1, V2 and V3, respectively. The maximum load value increases $21 \%$ from case V1 to V2 and $37 \%$ from case V1 to V3. The displacement values at maximum load are $1.56,1.48$ and $2.0 \mathrm{~mm}$.

The stress-strain curves for each displacement rate at $500{ }^{\circ} \mathrm{C}$ are presented in Fig. 9, as well as The stress-strain curve for the same material at room temperature for a nominal strain rate of $1.0 \cdot 10^{-3} \mathrm{~s}^{-1}$. 40 This room temperature curve was obtained from a homogeneous tensile test [41]. From the beginning of the deformation process, the three curves at $500{ }^{\circ} \mathrm{C}$ show the positive strain rate sensitivity. The negative temperature sensitivity is also clear when these curves are compared with the one at room temperature. The comparison of the room temperature curve with its homologous at $500{ }^{\circ} \mathrm{C}$ shows a decrease of almost $45 \%$ in the stress value. A dependence on the temperature is also observed for the elastic regime. Concerning Young's modulus, an average value of $106 \mathrm{GPa}$ is determined at $500{ }^{\circ} \mathrm{C}$ using the three curves presented in Fig. 9, whereas for room temperature, the Young's modulus is $185 \mathrm{GPa}$. 




Figure 8: Load-displacement $\left(\mathrm{u}_{x}\right)$ curves for the three displacement rates: $0.006,0.06$ and $0.6 \mathrm{~mm} \mathrm{~s}^{-1}$, which correspond to the tests V1, V2 and V3, respectively. The difference between the curves shows the positive sensitivity of the material to strain rate.

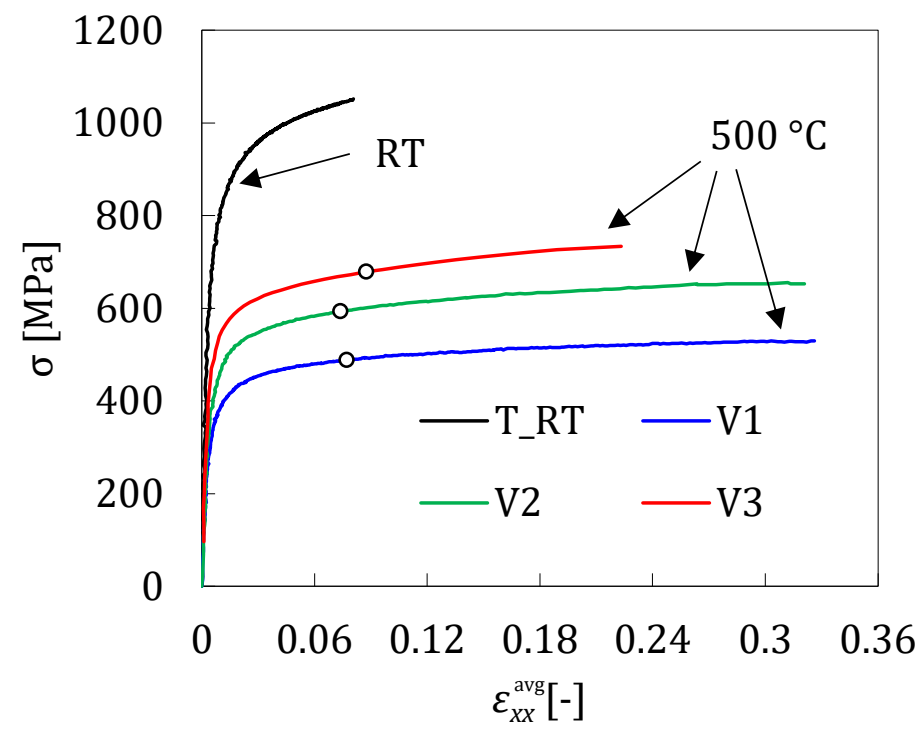

Figure 9: Stress-strain curves computed for a central section of the specimens. The colour lines correspond to the temperature of $500^{\circ} \mathrm{C}$. The black curve (T_RT) corresponds to room temperature (RT) obtained from a homogeneous tensile test [4].

\subsubsection{Subset and step size analysis}

During the tensile tests, the temperature gradient shown in Fig. 5 triggers a heterogeneous deformation process. The evolution of this process ends with strain localization at the centre of the specimen in a reduced region, which can be observed for the test V2 in Fig. 10. The selection of the parameters subset and step size of the DIC software are important in the accuracy of these measurements. A large subset size can mitigate 


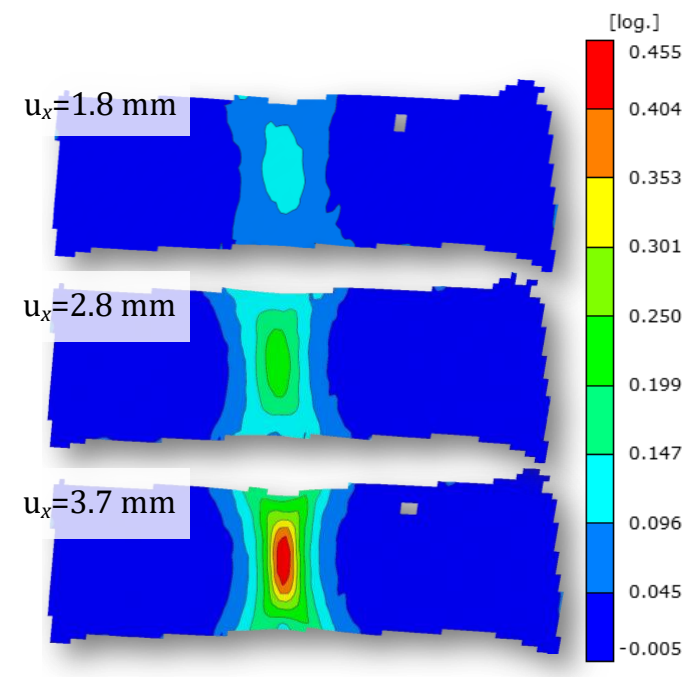

Figure 10: Major strain distribution for the test V2 at three different displacement points. The evolution of the deformation process is characterised by strain localization at the centre of the specimen.

the impact of noise in the strain measurements, but due to spatial smoothing, the maximum values can be underestimated. The step size controls the number of data points, and a small step size can be used to capture rapid changes in the peak values of the strain field. The results that supported the selection of the subset size of $20 \times 20$ and step size of 12 (Table 1) are briefly presented here. Three subset sizes $(15 \times 15$, $20 \times 20$ and $25 \times 25 \mathrm{px}^{2}$ ) and two step sizes $(7$ and $15 \mathrm{px}$ ) are selected, and a total of 6 sets of parameters are evaluated. This analysis is performed for the test V2. First, the results for the maximum load value are analysed and then the results for the instant before rupture.

Fig. 11a shows the distribution of the major strain for the maximum load value along the mid-plane



(a)



(b)

Figure 11: Subset and step size analysis for the test V2 at the maximum load value $\left(\mathrm{u}_{x}=1.48 \mathrm{~mm}\right.$, Fig. 8). Major strain evolution (a) for the total length of the ROI and (b) for a reduced length ( $x=-6 \mathrm{~mm}$ to $x=6 \mathrm{~mm}$ ). The reference set $20 \times 20 \times 12$ is represented by the solid black line. The remaining sets compose the sensitivity analysis and are represented by dashed lines. 
$y=0 \mathrm{~mm}$ of the ROI. In terms of shape of the strain distribution and maximum strain values, the different sets of parameters show similar results. The exception is the set composed by the subset $15 \times 15 \mathrm{px}^{2}$ and step size $7 \mathrm{px}$, which presents considerable oscillations. Fig. $11 \mathrm{~b}$ focus a smaller area, between $x=-6 \mathrm{~mm}$ and $x=6 \mathrm{~mm}$, and shows that the decrease of the step size increases the oscillations in the measurement results. Comparatively, the results for the step size of $12 \mathrm{px}$ show smooth responses without deterioration of the maximum strain values, hence any of these sets could be selected.

Fig. 12 presents the results for the instant before rupture. A higher strain value is captured with the step

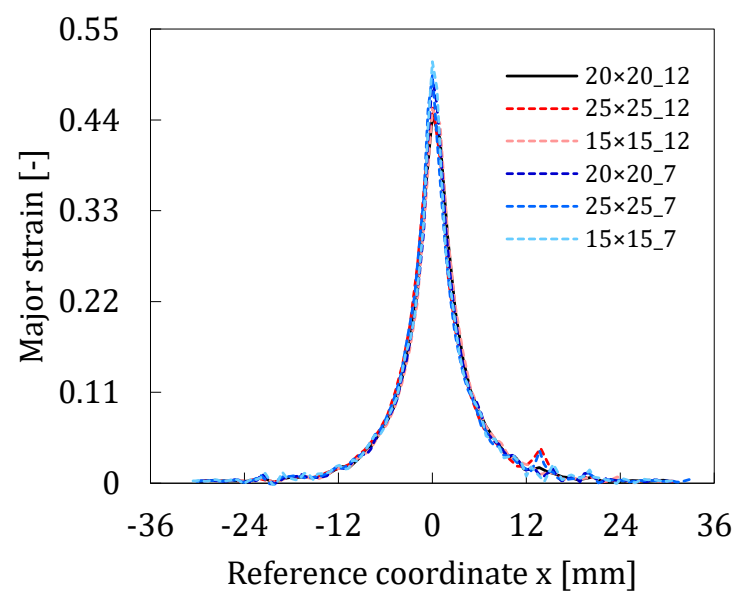

(a)

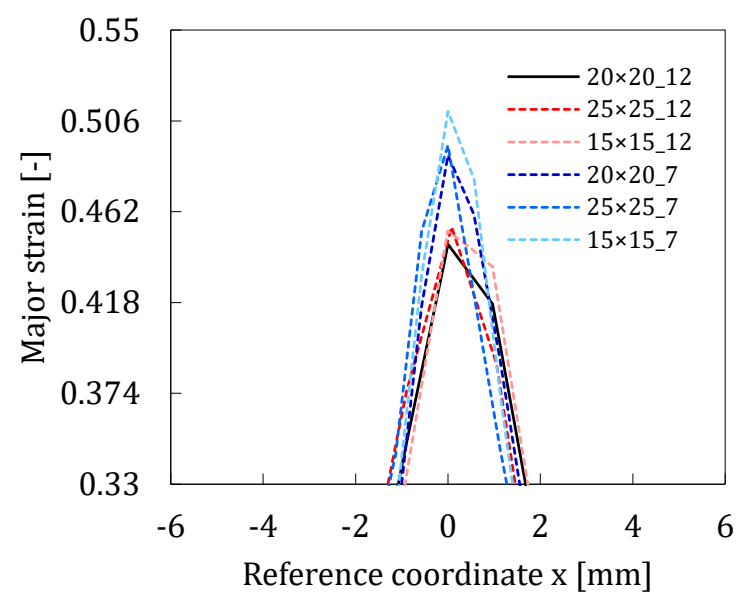

(b)

Figure 12: Subset and step size analysis for the test V2 at the instant before rupture. Major strain evolution (a) for the total length of the ROI and (b) for a reduced length $(x=-6 \mathrm{~mm}$ to $x=6 \mathrm{~mm})$. The reference set $20 \times 20 \times 12$ is represented by the solid black line. The remaining sets compose the sensitivity analysis and are represented by dashed lines.

size of $7 \mathrm{px}$ when compared with the step size of $12 \mathrm{px}$. In the worst scenario, the difference can be around $14 \%$.

The selection of $20 \times 20 \mathrm{px}^{2}$ for the subset and $12 \mathrm{px}$ for the step size is a compromise between accuracy and computational cost. Nevertheless, it should be noted that for measurements after the maximum load value the amplitude of the strain value can be underestimated.

\subsubsection{Strain and strain rate fields}

The evolution of the major strain along the mid-plane $y=0 \mathrm{~mm}$ is presented in Fig. 13a,Fig. 13c and Fig. 13e for the ROI up to the maximum load. The maximum major strain value occurs at the centre of the specimen and a decreasing trend is observed from the centre to the ends of the ROI. The maximum values of major strains are $0.093,0.086$ and 0.109 for the tests V1, V2 and V3, respectively. Moreover, after a displacement of $0.8 \mathrm{~mm}$ the evolution of the strains field occurs mainly between $x=-15$ and $x=15 \mathrm{~mm}$. According to this observation, the range of temperature between $x=-15$ and $x=15 \mathrm{~mm}$ is 460 to $500{ }^{\circ} \mathrm{C}$. In Fig. 13b. Fig. 13d and Fig. 13f it is shown the major-minor strain distribution for the three tests at the instant of maximum load value. The different data points of each test are under uniaxial tension up to this instant, a tendency that is not observed after the maximum load value, as can be seen in Fig. 14. After the maximum load value, the stress state deviates from the uniaxial tension state towards plane strain.

Fig. 15 shows the evolution of the strain rate field $\left(\dot{\varepsilon}_{x x}\right)$ up to the maximum load value for the same mid-plane. The maximum values of strain rate are $4.13 \cdot 10^{-4}, 3.69 \cdot 10^{-3}$ and $4.16 \cdot 10^{-2} \mathrm{~s}^{-1}$. These are in the same order of magnitude as the nominal strain rate of each test. The heterogeneity of the tests provides a wide dispersion of points, with a spatial distribution similar to the strain field.

In conclusion, the three tests conducted for a DP980 compose the database to be used with VFM. It is 


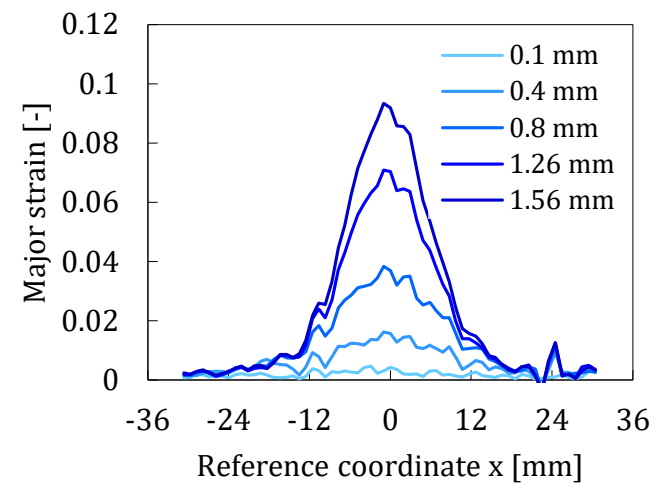

(a)

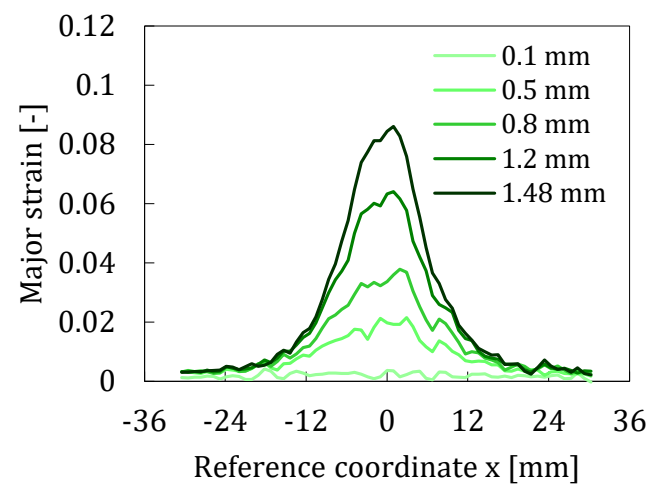

(c)



(e)

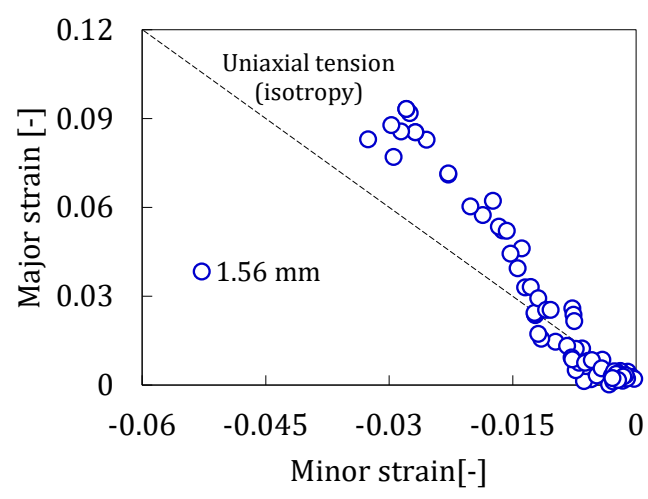

(b)



(d)

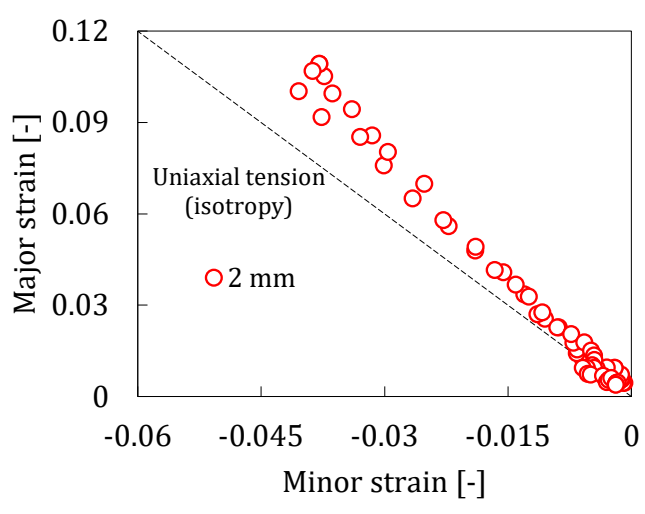

(f)

Figure 13: Major strain distribution and major-minor strain along the mid-plane of the specimen $(y=0 \mathrm{~mm})$ in the ROI, for the tests (a-b) V1, (c-d) V2 and (e-f) V3. The major strain distribution is compared for five different displacement values of each test up to the maximum load value. The major-minor strain plot refers to the maximum load value.

observed that the three tests provide a collection of points with different temperatures ranging from $360^{\circ} \mathrm{C}$ 


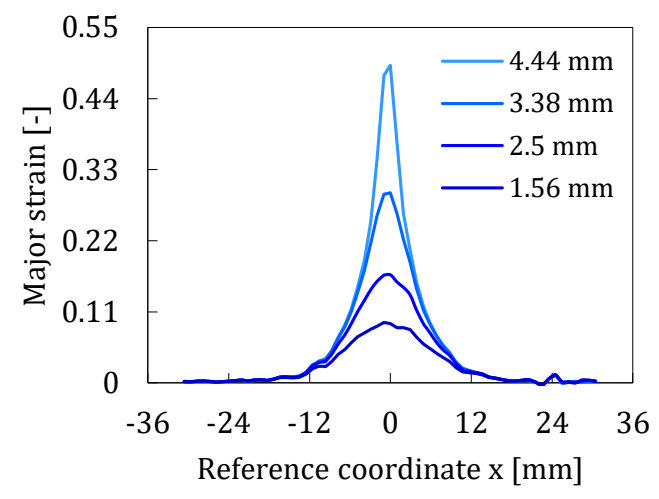

(a)

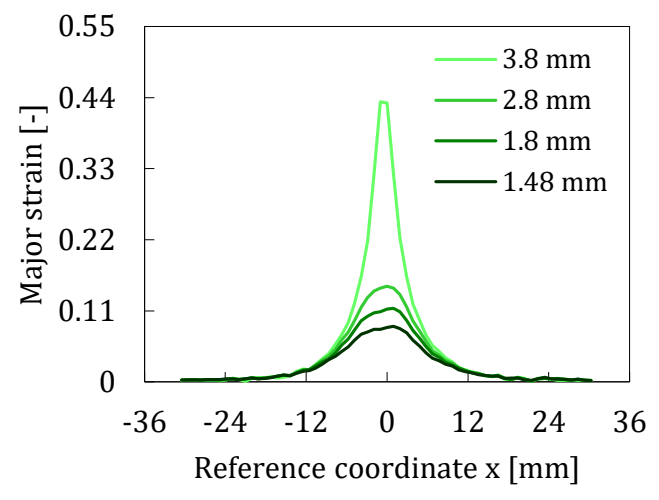

(c)

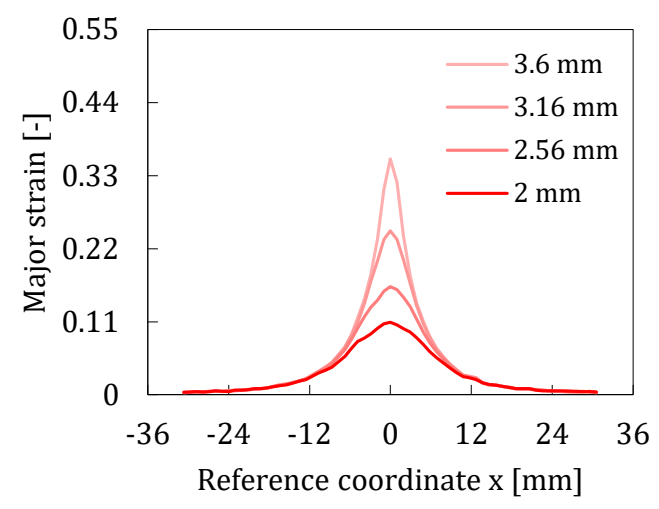

(e)

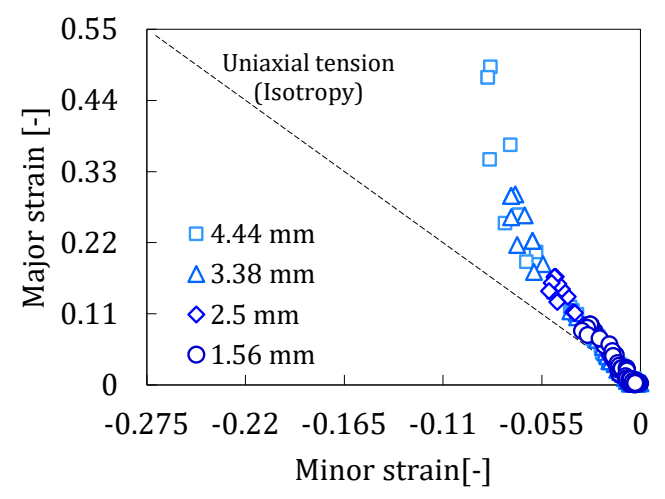

(b)

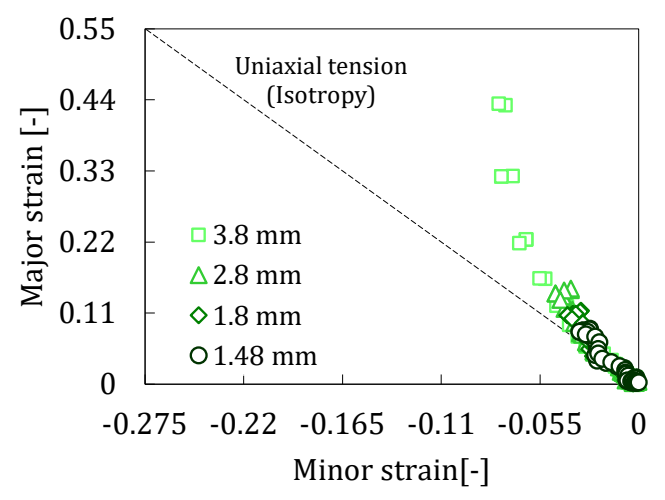

(d)

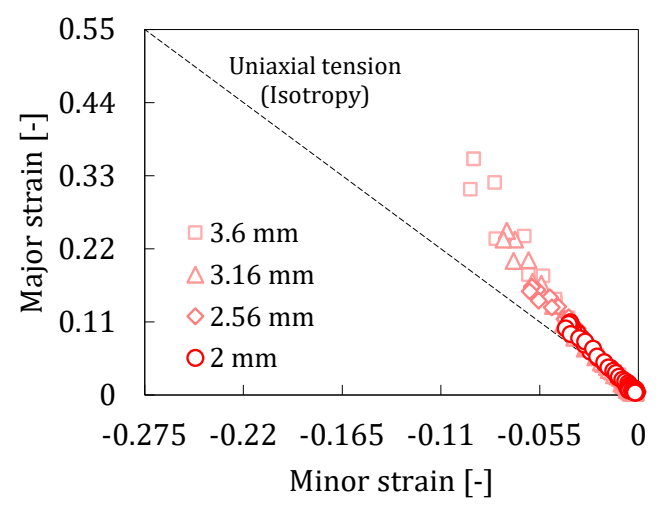

(f)

Figure 14: Major strain distribution and major-minor strain along the mid-plane of the specimen $(y=0 \mathrm{~mm})$ in the ROI, for the tests (a-b) V1, (c-d) V2 and (e-f) V3. Comparison for different displacement values after the maximum load value of each test.

to $500{ }^{\circ} \mathrm{C}$, for the ROI. The strain and strain rate fields present different magnitudes with the highest values 


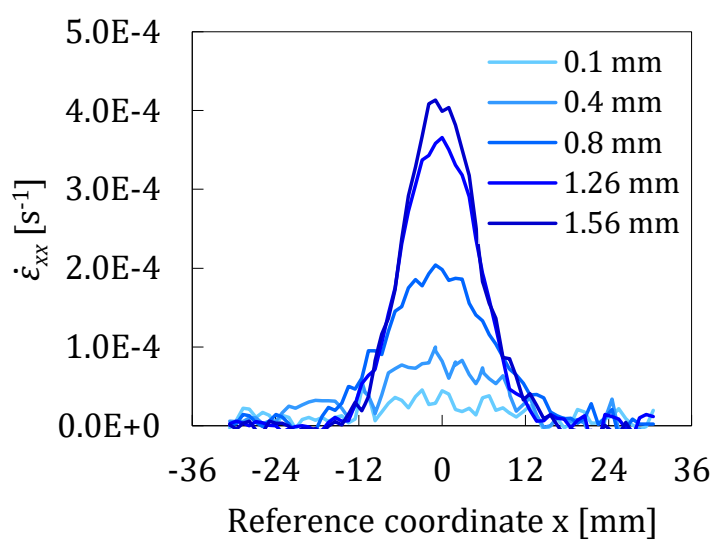

(a)

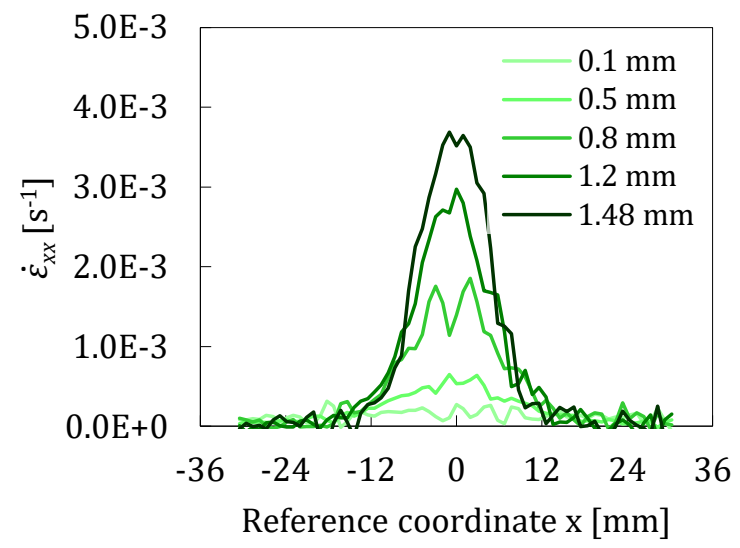

(b)

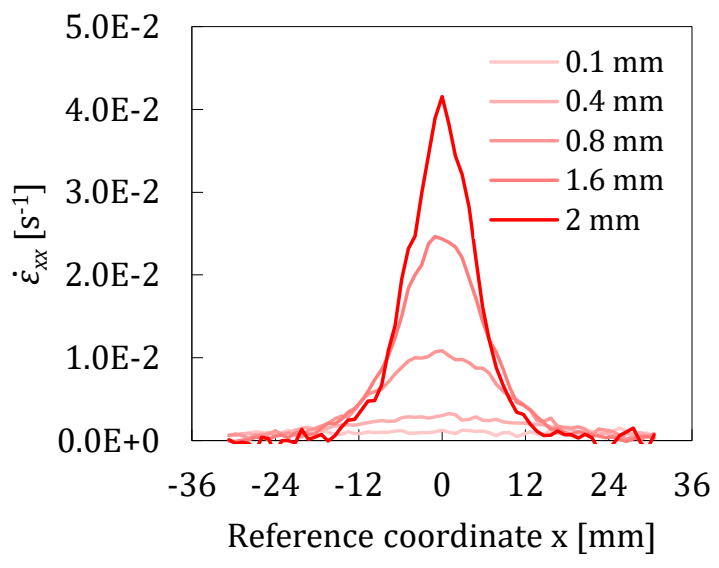

(c)

Figure 15: Strain rate $\left(\dot{\varepsilon}_{x x}\right)$ distribution along the mid-plane $y=0 \mathrm{~mm}$ in the ROI and, for the tests (a) V1, (b) V2 and (c) V3. The strain rate distribution is compared for five different displacement values of each test, including the instant of maximum load value.

at the centre of the specimen. The material has a positive sensitivity to strain rate and a negative sensitivity to temperature.

\subsection{Calibration results}

In this section, the calibration of the modified J-C model performed with the VFM and the experimental data from the heterogeneous thermo-mechanical tests is analysed. This analysis is divided into two parts. In the first part, the model is calibrated using a single test. The aim is to understand whether the calibration process and the chosen constitutive model lead to a good description of the thermo-mechanical behaviour of the material for the conditions of each test. In the second part, the three tests are used simultaneously in the calibration process. Thereby, the model is calibrated for a wider range of strain rates and consequently, for a vast range of information on the thermo-mechanical behaviour of the material.

Only data up to the maximum load value is used. As observed in the previous section, after the maximum 
load value the strain field measurements can be underestimated. Additionally, the 3D effects may become significant and the plane stress condition that is required for the stress computation in VFM can cause deviations in the internal virtual work computation [66, 67]. Therefore, for the VFM calibration, it is only considered the data up to the maximum load value.

A total of 7 parameters are identified, $\sigma_{0}, K, n, \sigma_{\mathrm{sat}}, C_{\mathrm{y}}, m$ and $C$, whereas the remaining parameters are considered known a priori: $\alpha=0.5, T_{\mathrm{tr}}=25^{\circ} \mathrm{C}, T_{\mathrm{m}}=1000^{\circ} \mathrm{C}$ and $\dot{\varepsilon}_{0}=1.0 \cdot 10^{-5} \mathrm{~s}^{-1}$. In the optimisation procedure, only one constraint is applied, which concerns the universe of solutions for each parameter. This constraint limits the admissible solutions to positive values only.

\subsubsection{Calibration with a single test}

The same initial set of parameters is used in the three calibrations. This set is characteristic of high strength steels, the exception being the parameter $\mathrm{C}$, which is attributed the zero value. The results of the identifications are presented in Table 2

The obtained parameters show significant differences from the initial set. This reveals that the objective

\begin{tabular}{ccccccccccc} 
& $\begin{array}{c}\sigma_{0} \\
{[\mathrm{MPa}]}\end{array}$ & $\begin{array}{c}K \\
{[\mathrm{MPa}]}\end{array}$ & $\begin{array}{c}n \\
{[-]}\end{array}$ & $\begin{array}{c}\sigma_{\text {sat }} \\
{[\mathrm{MPa}]}\end{array}$ & $\begin{array}{c}C_{\mathrm{y}} \\
{[-]}\end{array}$ & $\begin{array}{c}m \\
{[-]}\end{array}$ & $\begin{array}{c}C \\
{[-]}\end{array}$ & $\begin{array}{c}\varphi_{\text {ini }} \\
{[-] \cdot 10^{6}}\end{array}$ & $\begin{array}{c}\varphi_{\text {fin }} \\
{[-] \cdot 10^{6}}\end{array}$ & $\begin{array}{c}\text { Iter } \\
{[-]}\end{array}$ \\
\hline Initial & 600 & 900 & 0.060 & 1200 & 20 & 1.00 & 0.0 & - & - & - \\
V1 & 641 & 1044 & 0.033 & 1147 & 34 & 0.99 & $4.0 \cdot 10^{-8}$ & 1.2 & 0.4 & 28 \\
V2 & 697 & 1388 & 0.054 & 1060 & 53 & 1.10 & $1.3 \cdot 10^{-7}$ & 5.3 & 0.5 & 11 \\
V3 & 616 & 1637 & 0.068 & 997 & 69 & 1.17 & $2.6 \cdot 10^{-8}$ & 9.8 & 1.0 & 13 \\
\hline
\end{tabular}

Table 2: Results of the calibration using a single test. $\varphi_{\text {ini }}, \varphi_{\text {fin }}$ are the initial and final values of the objective function and Iter is the number of iterations in the Levenberg-Marquardt method.

function and experimental database are sensitive to the parameters. The exception is the parameter $C$, whose values are very close to zero, the initial value. This value close to zero means that the strain rate term (Eq. 4) in the modified J-C has a null impact.

The values of the objective function for the initial set $\left(\varphi_{\text {ini }}\right)$ and for the final set $\left(\varphi_{\text {fin }}\right)$ are also shown in The number of iterations for the test V1 is higher than for the other two tests. Fig. 16 shows the evolution of the parameters and objective function value for the three cases during the calibration process. There are differences in the number of iterations between the three cases, but the process is stable and almost reaches stabilised values after 60 evaluations.

$325 \quad$ Fig. 17 presents a comparison between internal and external virtual works. The internal virtual work is computed for the first virtual field $\mathbf{U}_{1}^{*}$ and using the initial (IVW_ini) and the finial (IVW_fin) sets of parameters. The external virtual work is computed using the measured load. The difference between initial and final solutions is noticeable, which results from the significant difference between the initial and final parameter sets. The final solutions provide a very good match between internal and external virtual works.

330 In Fig. 18 each term of the modified J-C model is individually plotted for the initial set and the final sets of parameters. Fig. 18a shows the evolution of the strain-hardening term (Eq. 2) with the increase of the equivalent plastic strain $\left(\bar{\varepsilon}^{\mathrm{p}}\right)$. The obtained parameters lead to distinct strain-hardening curves, which, in terms of stress values, are ordered according to the displacement rate of the test used in the calibration. Hence, the highest stress values are found in the strain-hardening curve obtained with test V3. It has to according to the displacement rate of the test used in the identification. Nevertheless, the three curves present similar shapes, high strain-hardening rates for low values of equivalent plastic deformation and a stress saturation value for high values of equivalent plastic deformation.

Fig. $18 \mathrm{~b}$ presents the temperature term (Eq. 3) evolution up to $500{ }^{\circ} \mathrm{C}$. This term shows a considerable 


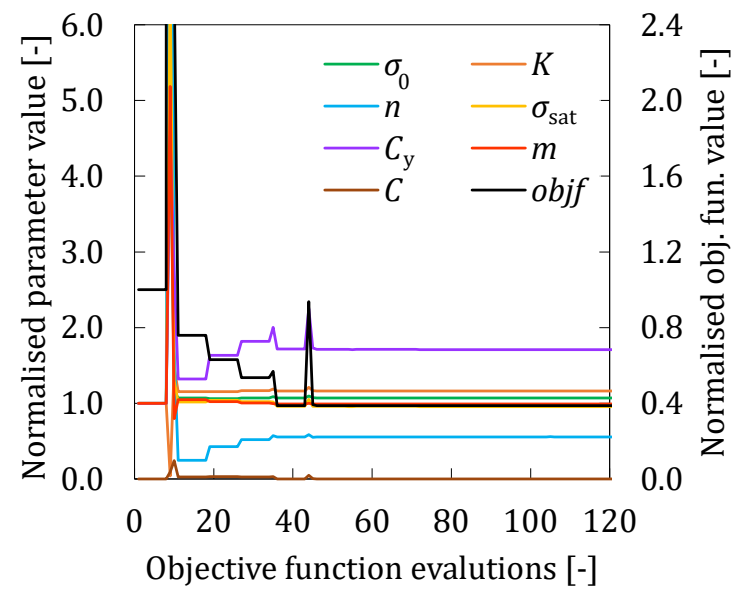

(a)

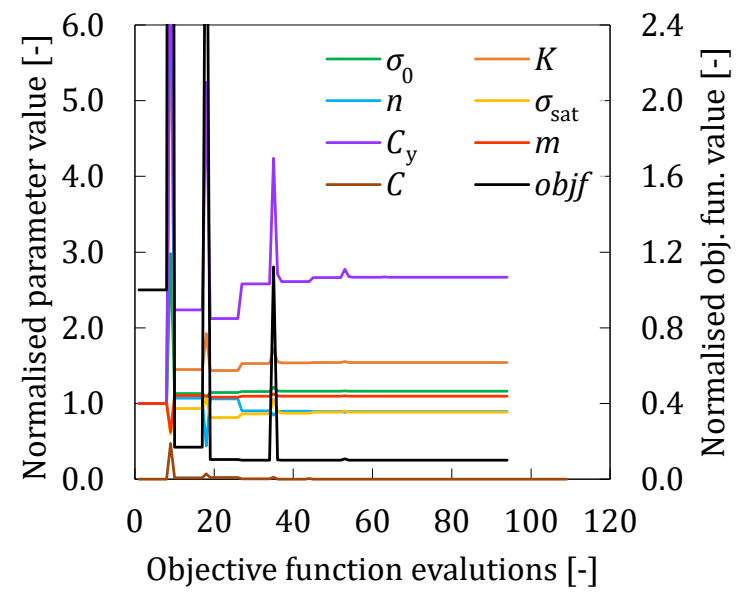

(b)

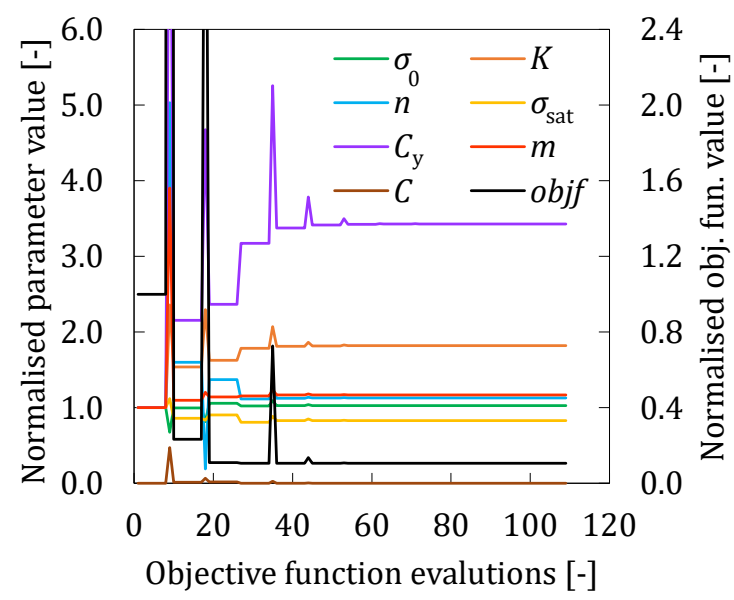

(c)

Figure 16: Evolution of the material parameters and objective function value (objf) during the calibration process: (a) V1, (b) V2 and (c) V3. The value of the parameters and objective function are normalised by the initial value, except for the case of the parameter $C$.

at $500{ }^{\circ} \mathrm{C}$, which means that the stress values are reduced to almost an half with an increase of temperature from 25 to $500^{\circ} \mathrm{C}$. As shown in Fig. 9, the difference between the stress-strain curve at room temperature curve and its homologous at $500{ }^{\circ} \mathrm{C}$ is about $45 \%$, which means that even without the information on the mechanical behaviour of the material at room temperature in the experimental database, the obtained solutions capture comparable stress values at room temperature. Moreover, similarly to the strain-hardening term, the impact of this term is ordered according to the displacement rate of the test used in the calibration. As mentioned before, the obtained values for the parameter $C$ are almost zero, thus the strain rate term (Eq. (4) becomes equal to 1.0, as shown in Fig. 18c. From these results, it can be concluded that the range of strain rates found in each test is not sufficient to activate this term, and the positive strain rate effect observed in Fig. 8 is not captured in any of the calibrations with a single test. Note that a different choice of the initial value for the parameter $C$ would lead to a different solution for the remaining parameters, but the sensitivity to $C$ would not change and the positive strain rate effect would be conditioned by this initial 


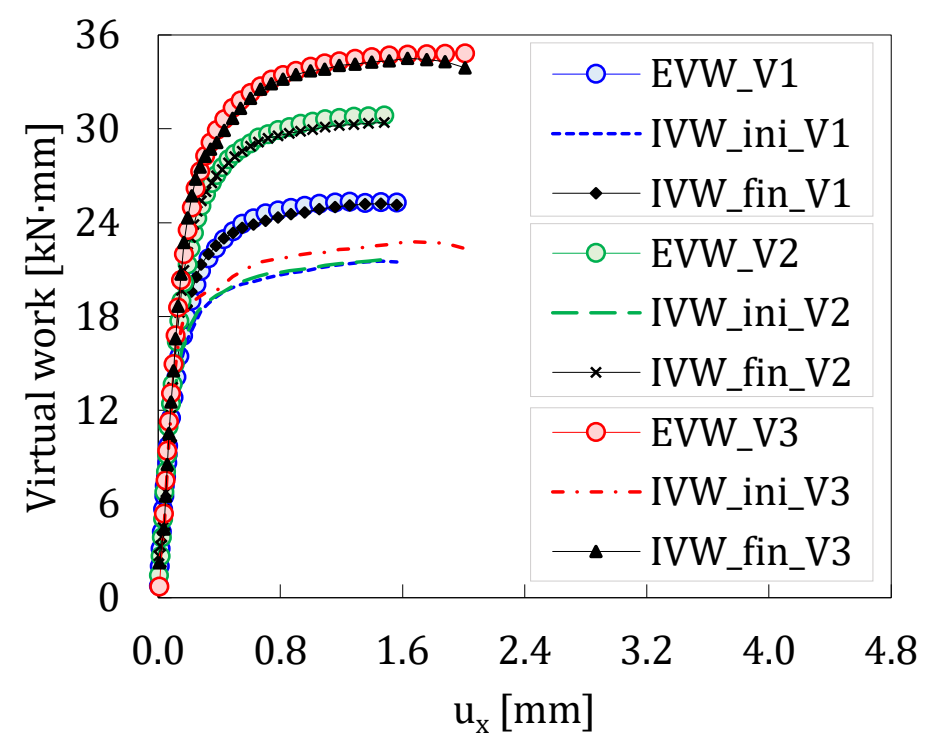

Figure 17: Internal virtual work (IVW) vs external virtual work (EVW). The IVW is plotted for the initial (IVW_ini) and final (IVW_fin) sets of parameters (Table 2 using the first virtual field $\mathbf{U}_{1}^{*}$. IVW_ini are represented by dashed lines, whereas IVW_fin are represented with solid lines and markers.

value.

Fig. 19 presents the strain-hardening term multiplied by the temperature term at $500{ }^{\circ} \mathrm{C}$. As the predominant stress state is uniaxial tension in the three tests, Fig. 19 and Fig. 9 can be compared. The level of flow stress for each curve is similar to the level of stress for the respective counterpart in Fig. 9 . According to this result, the magnitude of the flow stress for each test seems to be well described by the respective set of parameters.

To analyse the validity of the final sets of parameters presented in Table 2, these are tested as input data for the simulation of the heterogeneous tests. The FE model described in section 3.3 is used here. The predicted numerical load and strain distribution for the mid-plane of specimen (blue line in Fig. 7) are compared with the experimental counterparts. It is expected that the parameters give a good representation of the test that provided the information for the calibration. Moreover, it is included in these simulations the time steps beyond the maximum load value to evaluate the accuracy of the parameters when the results are extrapolated.

Fig. 20 presents the comparison of the numerical and the experimental load curves for the three tests. The three sets of parameters provide reasonable results up to the maximum load value. The set of parameters obtained with test V3 (Fig. 20c) obtained the best approximation to the experimental load curve, even in the extrapolation domain. In the other two cases (Fig. 20a and Fig. 20b), there is a slight overestimation for the maximum load value that spreads to the extrapolation domain.

In Fig. 21, the results for the logarithmic strain in the x-direction are presented for the three tests. The experimental results are labelled as Exp and the numerical ones as Num. Mload and Rup represent the time instants of maximum load and the last instant before rupture (the last step of the simulation). The results for the test V3 (Fig. 21c) are the most accurate ones. In this case, the bell-shaped curve of the strain distribution is well predicted. In the extrapolation domain, the maximum strain value is overestimated and the strain localisation is more severe than in the experimental results. Regarding Fig. 21a and Fig. 21b the amplitude of the strain field is underestimated and in both cases, their distribution is more uniform than the experimental strain field, but the trend is well captured. In the extrapolation domain, for both cases V1 and V2, the strain amplitude at the centre of the specimen is overestimated. 


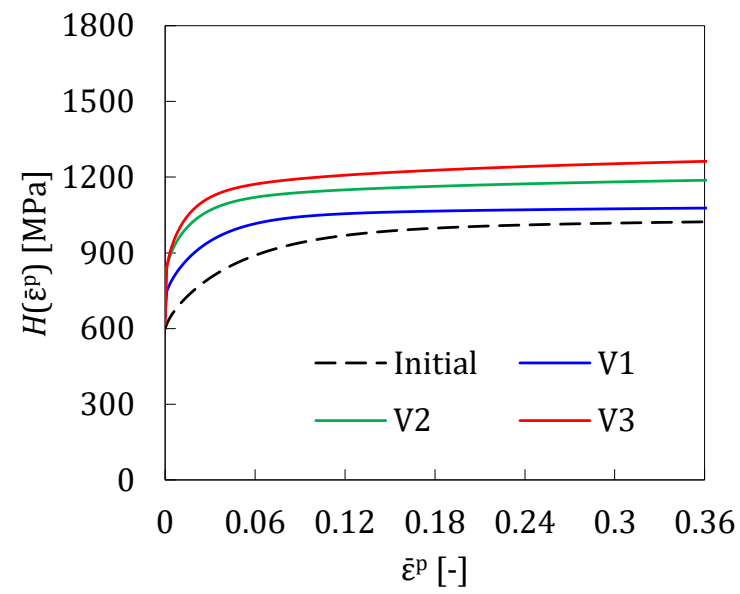

(a)

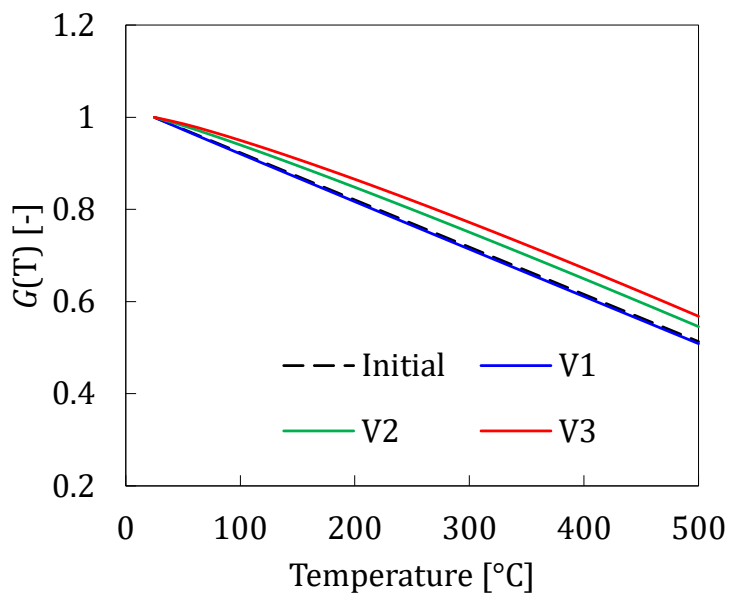

(b)



(c)

Figure 18: Evolution of three terms that compose the modified J-C model for the obtained sets of parameters presented in Table 2 (a) strain-hardening term (Eq. 2), (b) temperature term (Eq. 3) and (c) strain rate term (Eq. 4 .

\subsubsection{Calibration with multiple tests}

In this part, the results for the calibration of the modified J-C model using the three tests simultaneously are presented. Here, the goal is to reach a set of parameters that enables the representation of the conditions of the three tests through the modified J-C model. This is a more demanding problem than the previous one because the strain rate range is enlarged by the inclusion of the three tests in the same experimental database and the model is forced to capture the positive strain rate sensitivity of the material.

The results for a single initial set are presented in Table 3 . Note that the initial set is different from one presented in Table 2. This difference comes from the non-zero value attributed to $C$, which required to manually adjusted the remaining parameters to have an initial solution with realistic predictions of internal virtual work. All the parameters are activated in the optimisation and the initial value of the objective function decreased 54\%. The number of iterations for the optimisation Levenberg-Marquardt method is 12 . The evolution of the parameters and objective function value along the calibration is presented in Fig. 22 . The evolution of the material parameters seems more stable for the beginning of the optimisation than in 


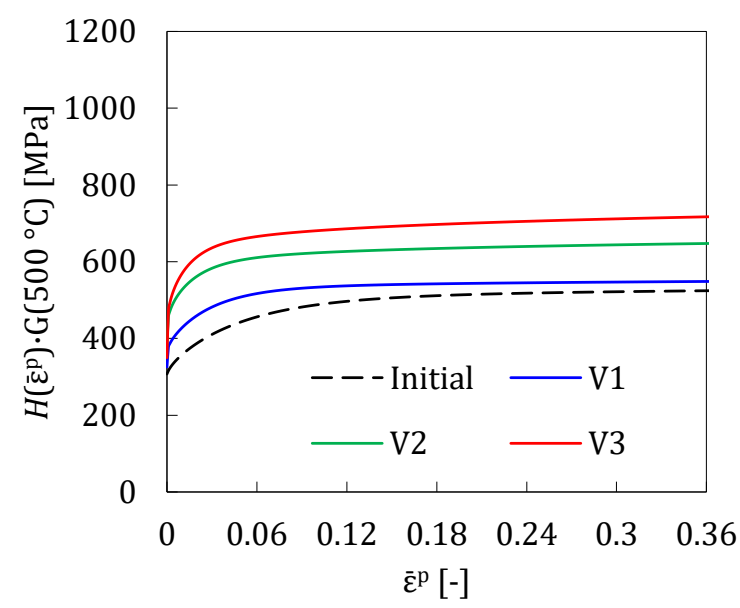

Figure 19: Evolution of the strain-hardening term (Eq. 3) affected by the temperature term (Eq. 3 at $500{ }^{\circ} \mathrm{C}$ for the final sets of parameters presented in Table 2

\begin{tabular}{ccccccccccc} 
& $\begin{array}{c}\sigma_{0} \\
{[\mathrm{MPa}]}\end{array}$ & $\begin{array}{c}K \\
{[\mathrm{MPa}]}\end{array}$ & $\begin{array}{c}n \\
{[-]}\end{array}$ & $\begin{array}{c}\sigma_{\text {sat }} \\
{[\mathrm{MPa}]}\end{array}$ & $\begin{array}{c}C_{\mathrm{y}} \\
{[-]}\end{array}$ & $\begin{array}{c}m \\
{[-]}\end{array}$ & $\begin{array}{c}C \\
{[-]}\end{array}$ & $\begin{array}{c}\varphi_{\text {ini }} \\
{[-] \cdot 10^{6}}\end{array}$ & $\begin{array}{c}\varphi_{\text {fin }} \\
{[-] \cdot 10^{6}}\end{array}$ & $\begin{array}{c}\text { Iter } \\
{[-]}\end{array}$ \\
\hline Initial & 327 & 966 & 0.070 & 513 & 81 & 1.19 & 0.11 & - & - & - \\
Final & 342 & 924 & 0.017 & 603 & 131 & 1.27 & 0.07 & 1.7 & 0.8 & 12 \\
\hline
\end{tabular}

Table 3: Results of the calibration with three tests. $\varphi_{\text {ini }}, \varphi_{\text {fin }}$ are the initial and final of values of the objective function and Iter is the number of iterations in the Levenberg-Marquardt method.

the previous cases with a single test.

Fig. 23 shows a comparison between internal and external virtual works. The internal virtual work is computed for the first virtual field $\mathbf{U}_{1}^{*}$ and using the initial (IVW_ini) and the final (IVW_fin) parameters set. The external virtual work is computed from the measured load. An underestimation of the IVW_fin for V2 is observed, as well as a slight underestimation for V3 in the final time steps. These two deviations are not observed in Fig. 17.

Fig. 24 shows the individual plot of the terms of the modified J-C model. In this case, the strain-hardening term (Fig. 24a) converges quickly to the stress saturation value, a consequence of the low value of the parameter $n$ and the high value of the parameter $C_{\mathrm{y}}$. The impact of the temperature continues to be significant (Fig. 24b), and the strain rate has now a relevant role (Fig. 24c). Since the strain-hardening term converges to the saturation value very quickly, the strain rate term assumes the leading role in the hardening process, imposing the increase in the flow stress through the positive effect of strain rate.

405 Similar to the previous section, the validity of the obtained set of parameters is tested in the numerical simulation of the three tests. Fig. 25 presents the results for the load prediction. From these figures, it is possible to conclude that parameter $C$ captures the positive strain rate effect. However, the results for the tests V1 (Fig. 25a) and V2 (Fig. 25b) are overestimated. In the case of test V3, the load is reasonably well predicted with a slight overestimation for higher displacement values (Fig. 25c).

${ }_{410}$ In terms of strain field, the results presented in Fig. 26 are worse than in Fig. 21. The best results are achieved for the test V3 (Fig. 26c) but, as in the other two cases, there is an underestimation of the amplitude value of the strain field when compared with the experimental measurements. In the extrapolation domain, the numerical predictions for the three cases tend to underestimate the strain localisation and present much lower strain values at the centre of the specimen. 


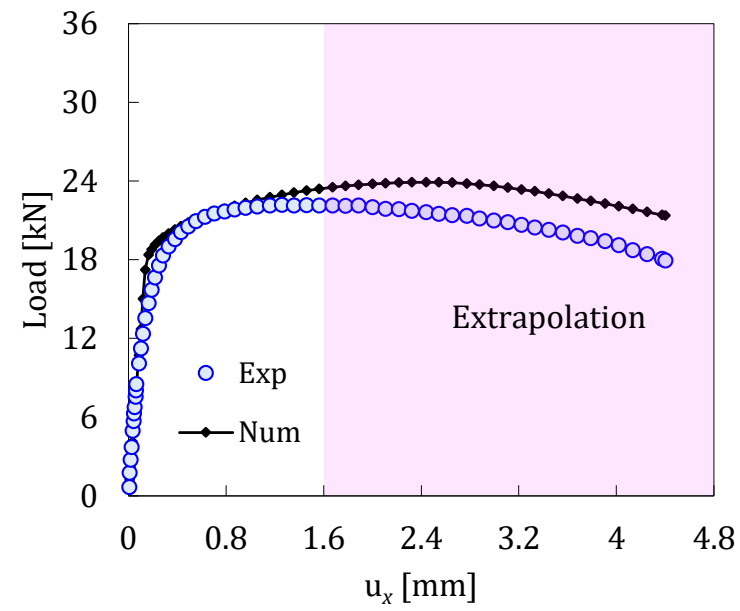

(a)

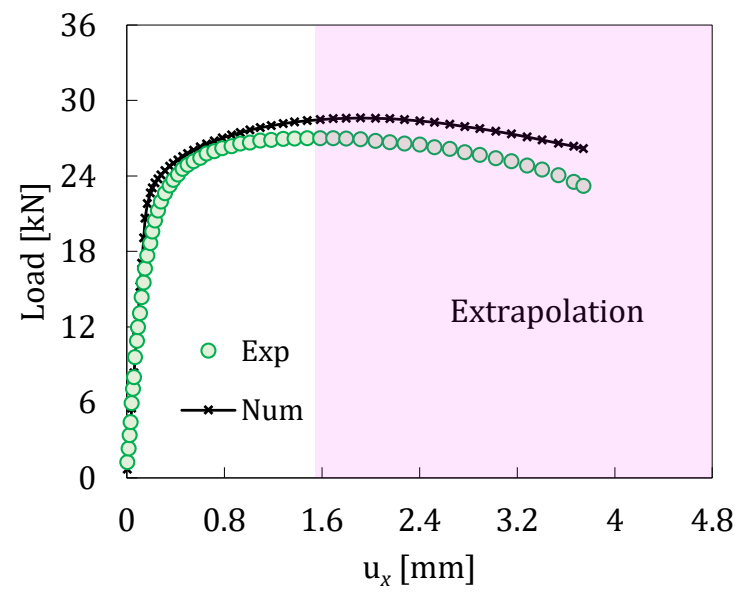

(b)



(c)

Figure 20: Comparison of experimental (Exp) and numerical (Num) load curves. The numerical load curves are obtained through FE analysis with the final sets of parameters presented in Table 2 (a) V1, (b) V2 and (c) V3.

\subsubsection{Discussion}

The calibration process with a single test resulted in three distinct parameters sets (Table 2). The validation of these sets shows a reasonably well prediction of the experimental load, which means that the stress level is well predicted. However, the obtained sets have almost zero values for the parameter $C$, which means that the strain rate effect is not captured by the strain rate term. The results suggest that the effect of strain rate is captured by the other two terms, which explains the significant differences in the obtained parameters from test to test. Consequently, the obtained sets of parameters have a limited applicability domain that is restricted to the conditions of the test used in the calibration process. Furthermore, for the test with the highest displacement rate (V3), the predicted strain field is in very good agreement with the experimental measurements but for the other two cases, the amplitude of the strain field is underestimated role in the strain distribution, and these results suggest that the effect of the temperature term is not well predicted for the tests with the lowest strain rates. 




(a)

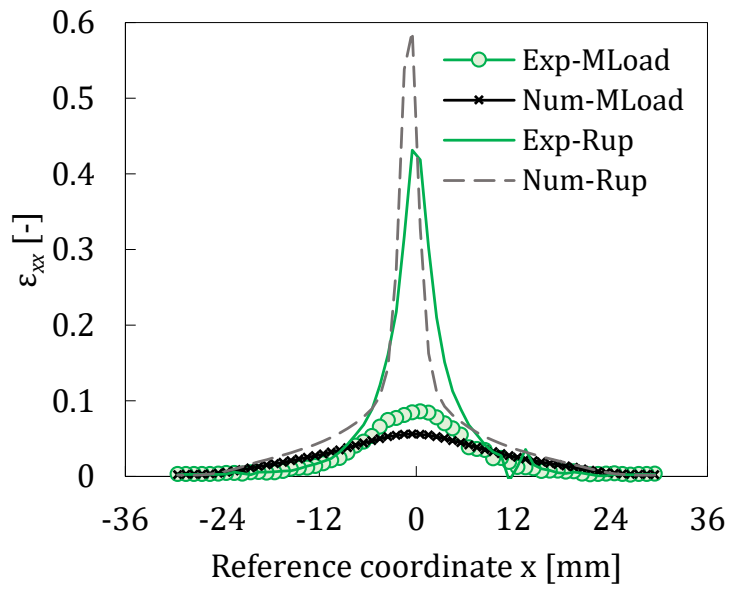

(b)



(c)

Figure 21: Comparison of experimental (Exp) and numerical (Num) spatial distribution of the xx component of the logarithmic strain $\left(\varepsilon_{x x}\right)$ along the reference $\mathrm{x}$-direction. The numerical results are obtained through FE analysis with the sets of parameters presented in Table 2 (a) V1, (b) V2 and (c) V3. Mload and Rup represent the time instants of maximum load and the last instant before rupture.

In the second part of this section, the three tests were combined in a single database to calibrate the modified J-C model. The results show that three tests bring sufficient information to calibrate the parameter $C$, which is not possible with a single test. In this case, the positive strain rate effect is captured by the strain rate term. Nevertheless, the results show the model's lack of flexibility to fit the experimental data provided by the three tests. This is observed in the results of the internal virtual work computation at the end of the optimisation (Fig. 23). Moreover, there is a significant deterioration of the strain field prediction, when compared with the previous results. This can be explained by the high impact of the strain rate term that brings a positive effect on the flow stress and balances the negative effect of the temperature term. Consequently, the localisation of the deformation at the centre of the specimen is attenuated and more uniform distributions of the strain field are obtained.

Regarding the optimisation process, just one set of initial parameters is presented in both analyses. Never- 




Figure 22: Evolution of the material parameters and objective function value (objf) during the calibration process for the experimental database composed by three tests. The values of the parameters and objective function are normalised by the initial value.

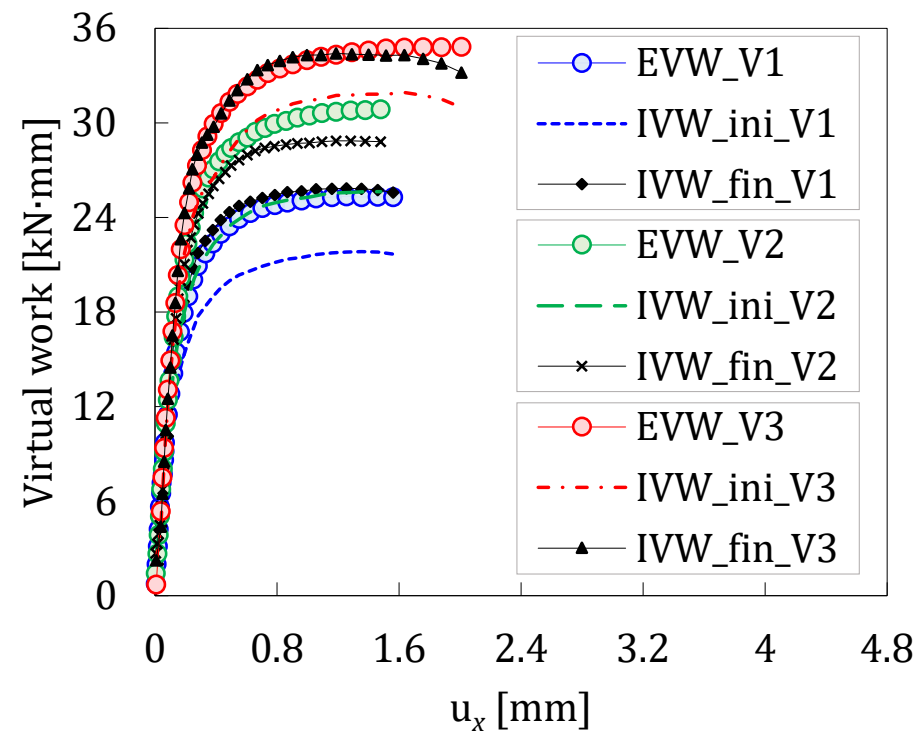

Figure 23: Internal virtual work (IVW) vs external virtual work (EVW). The IVW is plotted for the initial (IVW_ini) and final (IVW_fin) sets of parameters using the first virtual $\mathbf{U}_{1}^{*}$. IVW_ini are represented by dashed lines, whereas IVW_fin are represented with solid lines and markers.

theless, it was observed that the problem is sensitive to the initial set of parameters and multiple solutions are found. This reveals that the experimental database lacks information for some of the parameters. There are two potential solutions: reduce the parametrisation of the model, or improve the experimental database with more tests. Regarding the first solution, some parameters that have a specific meaning, like $\sigma_{0}$ that represents the initial yield stress, can be identified directly from a simple homogeneous tensile test. This helps to reduce the number of variables in the optimisation process and to mitigate the problem of multiple solutions. Regarding the second solution, including more tests in the database is also the path to more accurate results and to expand the validity of the obtained parameters. Moreover, the lack of flexibility of 




(a)

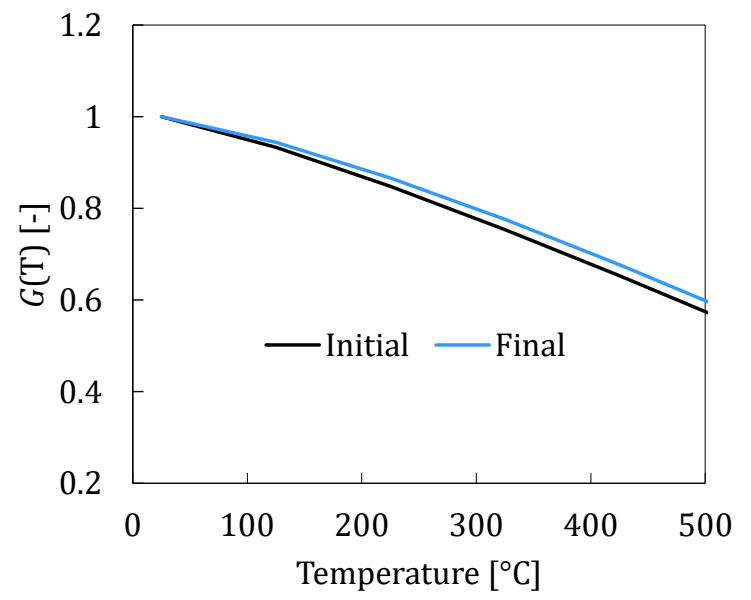

(b)

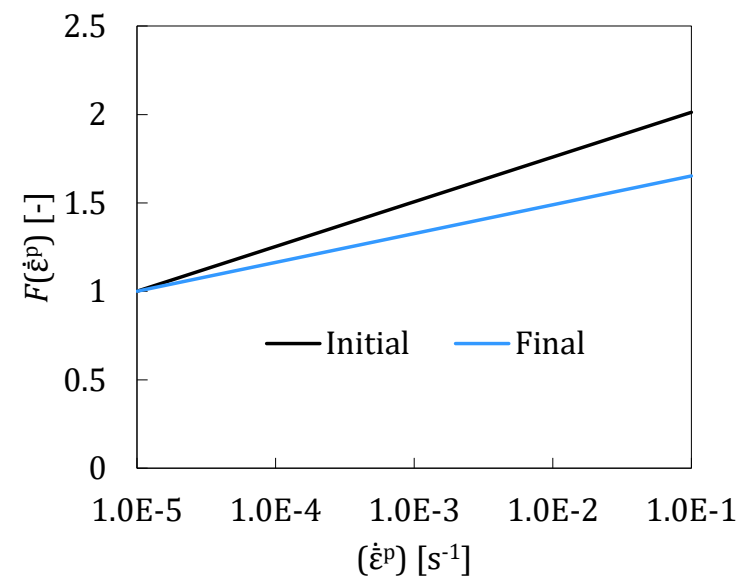

(c)

Figure 24: Evolution of three terms that compose the modified J-C model for the final set of parameters presented in Table 3 (a) strain-hardening term (Eq. 2), (b) temperature term (Eq. 3) and (c) strain rate term (Eq. 44.

the modified J-C model implies that more parameters are required to predict the mechanical behaviour of this material, thus it is reasonable to explore this solution.

The obtained results give some indications that the chosen model needs to be enhanced to accurately describe the mechanical behaviour of the DP980 under such temperature and strain rate conditions. The actual formulation of the J-C model can be a limitation. This formulation is usually called decoupled formulation because each term corresponds to an isolated effect. However, coupled formulations in which the different effects interact with each other may be more suitable for the material and conditions under study. The results for the calibrations with a single test (Table2) show that all the parameters of the model could depend on the strain rate effect, and also show a monotonic evolution for almost all parameters (except for the parameter $\sigma_{0}$ ). Therefore, the parameters $n$ and $C_{\mathrm{y}}$ could be a function of the strain rate effect. In this case, a coupled formulation would be required.

In sum, the VFM combined with this thermo-mechanical heterogeneous test has proved to be an effective solution for the simultaneous calibration of the material parameters of thermo-elasto-viscoplasticity models. 


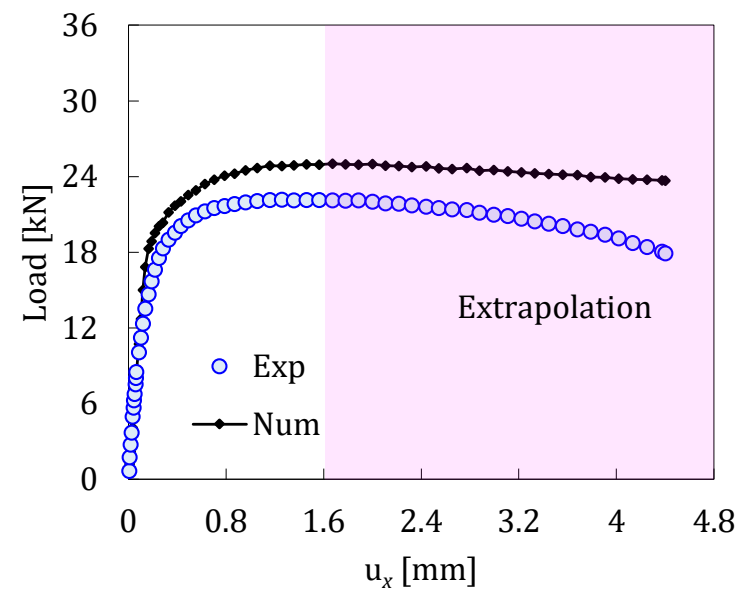

(a)

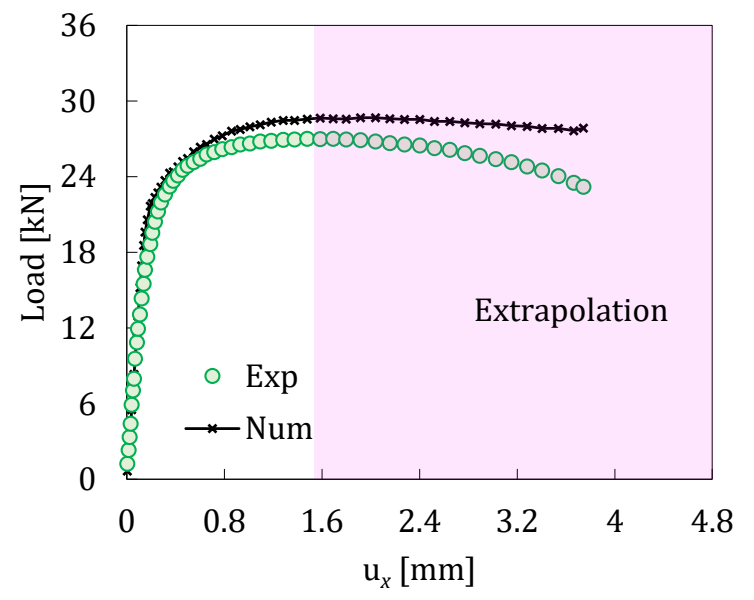

(b)

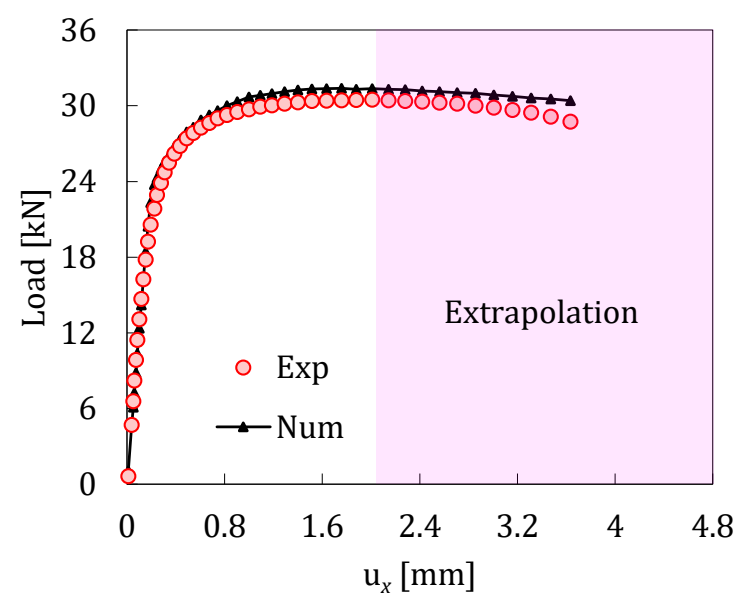

(c)

Figure 25: Comparison of experimental (Exp) and numerical (Num) load curves. The numerical load curves are obtained through FE analysis with the final set of parameters presented in Table 3 (a) V1, (b) V2 and (c) V3.

460

\begin{abstract}
of the material and due to the characteristic temperature field, it is a rather simple test to analyse and combine with VFM. As a result, this methodology benefits from the computational efficiency of VFM that is difficult to compare with other inverse strategies and from the simplicity of this heterogeneous thermomechanical test.
\end{abstract}

\section{Conclusions}

In this work, a novel calibration methodology for thermo-elasto-viscoplasticity models is presented as an alternative to classical calibration procedures that require a large number of homogeneous tensile tests. This methodology combines a thermo-mechanical heterogeneous test and the VFM, thus leveraging full-field data through the computational efficiency of VFM. To assess the feasibility of this methodology, a modified 




(a)



(b)



(c)

Figure 26: Comparison of experimental (Exp) and numerical (Num) spatial distribution of the xx component of the logarithmic strain $\left(\varepsilon_{x x}\right)$ along the reference $\mathrm{x}$-direction. The numerical results are obtained through $\mathrm{FE}$ analysis with the final set of parameters presented in Table 3. (a) V1, (b) V2 and (c) V3. Mload and Rup represent the time instants of maximum load and the last instant before rupture.

version of the J-C model is calibrated for the thermo-mechanical behaviour of DP980 steel.

Regarding the heterogeneous test, the following points can be highlighted:

- The test provides a large range of temperatures from $360{ }^{\circ} \mathrm{C}$ to $500{ }^{\circ} \mathrm{C}$, with a characteristic temperature field that can be captured by a $2^{\text {nd }}$ order polynomial function. Moreover, it can be assumed to be constant during the deformation process. These two characteristics simplify the analysis and the combination of the test with the VFM.

- The measured strain field has a bell-shaped distribution and reaches the highest values at the centre of the specimen. The strain rate field, which is a source of information for the calibration procedure, presents a similar shape to the strain field and up to the maximum load value, the magnitude of the strain rate field is in the same order of magnitude as the nominal strain rate. 
Regarding the calibration results, the analysis is divided into two parts. In the first part, the calibration with a single test performed at constant displacement is analysed. In the second part, the calibration is performed using the information of the three tests at different displacement rates. The following conclusions can be drawn:

- A experimental database with a single test leads to a calibrated model that predicts the thermomechanical behaviour of the material for the conditions of the test. Nevertheless, the information of a single test is insufficient to calibrate the strain rate term of the Johnson-Cook model and the positive strain rate sensitivity of the material is not captured, which limits the applicability of the model.

- The combination of three tests increases the range of strain rate values in the experimental database and consequently provides sufficient information to calibrate the three terms of the model. In this case, the material positive sensitivity to strain-rate is reasonably captured by the strain rate term.

In sum, this work shows that the proposed methodology reaches reasonable results and represents a potential alternative to classical procedures. Nevertheless, there is substantial room for improvement. For instance, the experimental database can be enhanced with different temperature ranges. The observed lack of flexibility of the modified J-C model suggests that a more complex model is required to describe the thermo-mechanical behaviour of DP980 steel. Therefore, in a future study, it is recommended to increase the information for different temperatures and strain rates and to consider a constitutive model with a coupled formulation. Additionally, it is recommended to perform a sensitivity analysis using synthetic image deformation 68, 69. This analysis will be important to understand the impact of noise on the methodology and, in the case of a more complex model, to identify which material parameters require more information for an accurate calibration [31, 70].

\section{Acknowledgements}

This project has received funding from the Research Fund for Coal and Steel under grant agreement No 888153. The authors also gratefully acknowledge the financial support of the Portuguese Foundation for Science and Technology (FCT) under the projects PTDC/EME-APL/29713/2017 (CENTRO-010145-FEDER-029713), PTDC/EME-EME/31243/2017 (POCI-01-0145-FEDER-031243) and PTDC/EMEEME/30592/2017 (POCI-01-0145-FEDER-030592) by UE/FEDER through the programs CENTRO 2020 and COMPETE 2020, and UID/EMS/00481/2013-FCT under CENTRO-01-0145-FEDER-022083. The authors also would like to acknowledge the Région Bretagne (France) for its financial support. J.M.P. Martins is also grateful to the FCT for the PhD grant SFRH/BD/117432/2016.

\section{References}

[1] H. Karbasian, A. Tekkaya, A review on hot stamping Journal of Materials Processing Technology 210 (15) (2010) 2103 2118. doi:https://doi.org/10.1016/j.jmatprotec.2010.07.019

URL http://www.sciencedirect.com/science/article/pii/S092401361000213X

[2] S. Toros, F. Ozturk, I. Kacar, Review of warm forming of aluminum-magnesium alloys Journal of Materials Processing Technology 207 (1) (2008) 1 -12. doi:https://doi.org/10.1016/j.jmatprotec.2008.03.057 URL http://www.sciencedirect.com/science/article/pii/S092401360800318X

[3] Z. Cai, M. Wan, Z. Liu, X. Wu, B. Ma, C. Cheng, Thermal-mechanical behaviors of dual-phase steel sheet under warmforming conditions International Journal of Mechanical Sciences 126 (2017) 79 - 94. doi:https://doi.org/10.1016/j. ijmecsci.2017.03.009

URL http://www.sciencedirect.com/science/article/pii/S0020740317305787

[4] A. K. Sachdev, J. E. Hunter, Thermal effects during uniaxial straining of steels, Metallurgical Transactions A 13 (6) (1982) 1063-1067.

[5] B. Erice and C.C. Roth and D. Mohr, Stress-state and strain-rate dependent ductile fracture of dual and complex phase steel Mechanics of Materials 116 (2018) 11 - 32, iUTAM Symposium on Dynamic Instabilities in Solids. doi:https: //doi.org/10.1016/j.mechmat.2017.07.020 URL http://www.sciencedirect.com/science/article/pii/S0167663616305361

[6] J. Sung, J. Kim, R. Wagoner, A plastic constitutive equation incorporating strain, strain-rate, and temperature, International Journal of Plasticity 26 (12) (2010) 1746 - 1771. doi:https://doi.org/10.1016/j.ijplas.2010.02.005 URL http://www.sciencedirect.com/science/article/pii/S0749641910000276 
530 [7] H. Kim, J. W. Yoon, K. Chung, M.-G. Lee, A multiplicative plastic hardening model in consideration of strain softening and strain rate: Theoretical derivation and characterization of model parameters with simple tension and creep test International Journal of Mechanical Sciences 187 (2020) 105913. doi:https://doi.org/10.1016/j.ijmecsci.2020.105913 URL https://www.sciencedirect.com/science/article/pii/S0020740320310985

[8] L. Gambirasio, E. Rizzi, On the calibration strategies of the Johnson-Cook strength model: Discussion and applications

535 to experimental data, Materials Science and Engineering: A 610 (2014) 370 - 413. doi:https://doi.org/10.1016/j. msea.2014.05.006 URL http://www.sciencedirect.com/science/article/pii/S0921509314005875

[9] E. Markiewicz, B. Langrand, D. Notta-Cuvier, A review of characterisation and parameters identification of materials constitutive and damage models: From normalised direct approach to most advanced inverse problem resolution International Journal of Impact Engineering 110 (2017) 371 - 381, special Issue in honor of Seventy Fifth Birthday of Professor N. K. Gupta. doi:https://doi.org/10.1016/j.ijimpeng.2017.01.028

URL http://www.sciencedirect.com/science/article/pii/S0734743X16307746

[10] Z. Shao, N. Li, J. Lin, T. Dean, Formability evaluation for sheet metals under hot stamping conditions by a novel biaxial testing system and a new materials model International Journal of Mechanical Sciences 120 (2017) $149-158$. doi:https://doi.org/10.1016/j.ijmecsci.2016.11.022 URL http://www.sciencedirect.com/science/article/pii/S002074031630892X

[11] A. Smith, Z. Chen, J. Lee, M. Lee, R. Wagoner, Effective method for fitting complex constitutive equations, International Journal of Plasticity 58 (2014) 100 - 119, in Honor of Kwansoo Chung. doi:https://doi.org/10.1016/j.ijplas.2014. 01.005

URL http://www.sciencedirect.com/science/article/pii/S074964191400014X

[12] G. Johnson, W. H. Cook, A constitutive model and data for metals subjected to large strains, high strain rates and high temperatures, in: Proceedings of the 7th International Symposium on Ballistics, Vol. 21, The Netherlands, 1983, pp. $541-547$.

[13] H. Laurent and J. Coër and P.Y. Manach and M.C. Oliveira and L.F. Menezes, Experimental and numerical studies on

555 the warm deep drawing of an Al-Mg alloy International Journal of Mechanical Sciences 93 (2015) 59 - 72. doi:https: //doi.org/10.1016/j.ijmecsci.2015.01.009

URL http://www.sciencedirect.com/science/article/pii/S0020740315000132

[14] F. Pierron, M. Grédiac, Towards material testing 2.0. a review of test design for identification of constitutive parameters

1. from full-field measurements Strain 57 (1) (2021) e12370, e12370 10.1111/str.12370. arXiv:https://onlinelibrary. wiley.com/doi/pdf/10.1111/str.12370 doi:https://doi.org/10.1111/str.12370

URL https://onlinelibrary.wiley.com/doi/abs/10.1111/str.12370

[15] M. A. Sutton, J. J. Orteu, H. Schreier, Image correlation for shape, motion and deformation measurements: basic concepts, theory and applications, Springer Science \& Business Media, 2009.

[16] S. Belhabib, H. Haddadi, M. Gaspérini, P. Vacher, Heterogeneous tensile test on elastoplastic metallic sheets: Comparison between fem simulations and full-field strain measurements International Journal of Mechanical Sciences 50 (1) (2008) 14-21. doi:https://doi.org/10.1016/j.ijmecsci.2007.05.009 URL https://www.sciencedirect.com/science/article/pii/S0020740307000860

[17] N. Souto, A. Andrade-Campos, S. Thuillier, A numerical methodology to design heterogeneous mechanical tests International Journal of Mechanical Sciences 107 (2016) 264-276. doi:https://doi.org/10.1016/j.ijmecsci.2016.01.021 URL https://www.sciencedirect.com/science/article/pii/S0020740316000278

[18] B. Barroqueiro, A. Andrade-Campos, J. D. de Oliveira, R. Valente, Design of mechanical heterogeneous specimens using topology optimization. International Journal of Mechanical Sciences 181 (2020) 105764. doi:https://doi.org/10.1016/ j.ijmecsci.2020.105764

URL https://www.sciencedirect.com/science/article/pii/S0020740320305166

575 [19] T. Pottier, F. Toussaint, H. Louche, P. Vacher, Inelastic heat fraction estimation from two successive mechanical and thermal analyses and full-field measurements European Journal of Mechanics - A/Solids 38 (2013) 1 - 11. doi:https: //doi.org/10.1016/j.euromechsol.2012.09.002 URL http://www.sciencedirect.com/science/article/pii/S0997753812001064

[20] S. Charlès, J.-B. Le Cam, Inverse identification of constitutive parameters from heat source fields: A local approach

580 applied to hyperelasticity Strain 56 (2) (2020) e12334, e12334 10.1111/str.12334. arXiv:https://onlinelibrary.wiley. com/doi/pdf/10.1111/str.12334 doi:https://doi.org/10.1111/str.12334

URL https://onlinelibrary.wiley.com/doi/abs/10.1111/str.12334

[21] S. Avril, M. Bonnet, A.-S. Bretelle, M. Grédiac, F. Hild, P. Ienny, F. Latourte, D. Lemosse, S. Pagano, E. Pagnacco, et al., Overview of identification methods of mechanical parameters based on full-field measurements, Experimental Mechanics 48 (4) (2008) 381.

[22] M. Grédiac, F. Hild, Full-field measurements and identification in solid mechanics, Wiley Online Library, 2013.

[23] H. Haddadi, S. Belhabib, Improving the characterization of a hardening law using digital image correlation over an

enhanced heterogeneous tensile test International Journal of Mechanical Sciences 62 (1) (2012) $47-56$. doi:https: //doi.org/10.1016/j.ijmecsci.2012.05.012

$590 \quad$ URL http://www.sciencedirect.com/science/article/pii/S0020740312001221

[24] Y. Chemisky, F. Meraghni, N. Bourgeois, S. Cornell, R. Echchorfi, E. Patoor, Analysis of the deformation paths and thermomechanical parameter identification of a shape memory alloy using digital image correlation over heterogeneous tests International Journal of Mechanical Sciences 96-97 (2015) 13 - 24. doi:https://doi.org/10.1016/j.ijmecsci. 2015.03 .007 
[25] F. Pierron, M. Grédiac, The virtual fields method: extracting constitutive mechanical parameters from full-field deformation measurements, Springer Science \& Business Media, 2012.

[26] L. Zhang, S. Thakku, M. Beotra, M. Baskaran, T. Aung, J. Goh, N. Strouthidis, M. Girard, Verification of a virtual fields method to extract the mechanical properties of human optic nerve head tissues in vivo, Biomechanics and Modeling in Mechanobiology 16 (3) (2017) 871-887.

[27] J.M.P. Martins, and A. Andrade-Campos, and S.Thuillier,, Comparison of inverse identification strategies for constitutive mechanical models using full-field measurements International Journal of Mechanical Sciences 145 (2018) 330 - 345. doi:https://doi.org/10.1016/j.ijmecsci.2018.07.013 URL http://www.sciencedirect.com/science/article/pii/S0020740318305836

$605[28]$ M. Grédiac, Principe des travaux virtuels et identification, Comptes rendus de l'Académie des sciences. Série 2, Mécanique, Physique, Chimie, Sciences de l'univers, Sciences de la Terre 309 (1) (1989) 1-5.

[29] A. Lattanzi, F. Barlat, F. Pierron, A. Marek, M. Rossi, Inverse identification strategies for the characterization of transformation-based anisotropic plasticity models with the non-linear VFM, International Journal of Mechanical Sciences 173 (2020) 105422. doi:https://doi.org/10.1016/j.ijmecsci.2020.105422 URL http://www.sciencedirect.com/science/article/pii/S0020740319328826

[30] A. Marek, and F.M. Davis, and J.-H. Kim, and F. Pierron,, Experimental validation of the sensitivity-based virtual fields for identification of anisotropic plasticity models, Experimental Mechanics (2020) 1-26.

[31] J. Fu, W. Xie, J. Zhou, L. Qi, A method for the simultaneous identification of anisotropic yield and hardening constitutive parameters for sheet metal forming, International Journal of Mechanical Sciences (2020) 105756

615 [32] S.N. Grama and S.J. Subramanian and F. Pierron, On the identifiability of Anand visco-plastic model parameters using the Virtual Fields Method Acta Materialia 86 (2015) 118 -136. doi:https://doi.org/10.1016/j.actamat.2014.11.052 URL http://www.sciencedirect.com/science/article/pii/S1359645414009057

[33] E.M.C. Jones, and J.D. Carroll, and K.N. Karlson, and S.L.B. Kramer, and R.B. Lehoucq, and P.L. Reu, and D.Z. Turner,, Parameter covariance and non-uniqueness in material model calibration using the Virtual Fields Method Computational Materials Science 152 (2018) 268-290. doi:https://doi.org/10.1016/j.commatsci.2018.05.037 URL http://www.sciencedirect.com/science/article/pii/S0927025618303501

[34] G. Valeri and B. Koohbor and A. Kidane and M.A. Sutton, , Determining the tensile response of materials at high

temperature using DIC and the Virtual Fields Method, Optics and Lasers in Engineering 91 (2017) 53 - 61. doi:https: //doi.org/10.1016/j.optlaseng.2016.11.004 URL http://www.sciencedirect.com/science/article/pii/S0143816616304109

[35] L. Rose, A. Menzel, Optimisation based material parameter identification using full field displacement and temperature measurements. Mechanics of Materials 145 (2020) 103292. doi:https://doi.org/10.1016/j.mechmat.2019.103292 URL https://www.sciencedirect.com/science/article/pii/S0167663619303199

[36] T. Archer, M. Berny, P. Beauchêne, F. Hild, Creep behavior identification of an environmental barrier coating using full-field measurements, Journal of the European Ceramic Society 40 (15) (2020) 5704-5718. doi:https://doi.org/10. $1016 / j \cdot$ jeurceramsoc.2020.06.009 URL https://www.sciencedirect.com/science/article/pii/S0955221920304593

[37] J. Coër, C. Bernard, H. Laurent, A. Andrade-Campos, S. Thuillier, The effect of temperature on anisotropy properties of an aluminium alloy, Experimental Mechanics 51 (7) (2011) 1185-1195.

635 [38] Z. Shao, N. Li, J. Lin, T. A. Dean, Strain measurement and error analysis in thermo-mechanical tensile tests of sheet metals for hot stamping applications, Proceedings of the Institution of Mechanical Engineers, Part C: Journal of Mechanical Engineering Science 232 (11) (2018) 1994-2008. arXiv:https://doi.org/10.1177/0954406217714011. doi:10.1177/0954406217714011

URL https://doi.org/10.1177/0954406217714011

[39] Y. Li, S. Li, Y. Chen, G. Han, Constitutive parameters identification based on dic assisted thermo-mechanical tensile test

for hot stamping of boron steel, Journal of Materials Processing Technology 271 (2019) 429-443. doi:https://doi.org/ $10.1016 / j \cdot j$ matprotec.2019.04.020 URL https://www.sciencedirect.com/science/article/pii/S0924013619301426

[40] R. Zhang, Z. Shao, J. Lin, T. Dean, Measurement and analysis of heterogeneous strain fields in uniaxial tensile tests for boron steel under hot stamping conditions, Experimental Mechanics 60 (9) (2020) 1289-1300.

[41] A. Mishra, Experimental investigation and numerical prediction of rupture in bending of metallic sheets, Ph.D. thesis, Lorient (2013).

[42] A. Mishra, S. Thuillier, Investigation of the rupture in tension and bending of DP980 steel sheet, International Journal of Mechanical Sciences 84 (2014) 171 -181. doi:https://doi.org/10.1016/j.ijmecsci.2014.04.023 URL http://www.sciencedirect.com/science/article/pii/S0020740314001581

$43]$ G. Aramis, Theory and user manual (2018).

[44] B. Pan, D. Wu, J. Gao, High-temperature strain measurement using active imaging digital image correlation and infrared radiation heating The Journal of Strain Analysis for Engineering Design 49 (4) (2014) 224-232. doi:10.1177/ 0309324713502201 URL https://doi.org/10.1177/0309324713502201

[45] N. Demazel, H. Laurent, J. Coër, M. Carin, P. Le Masson, J. Favero, R. Canivenc, H. Salmon-Legagneur, Investigation of the progressive hot die stamping of a complex boron steel part using numerical simulations and gleeble tests The International Journal of Advanced Manufacturing Technology 99 (1) (2018) 803-817. doi:10.1007/s00170-018-2532-6 URL https://doi.org/10.1007/s00170-018-2532-6 
[46] J. P. Martins, A. Andrade-Campos, S. Thuillier, Calibration of Johnson-Cook Model Using Heterogeneous ThermoMechanical Tests, Procedia Manufacturing 47 (2020) 881 - 888, 23rd International Conference on Material Forming. doi:https://doi.org/10.1016/j.promfg.2020.04.274 URL http://www.sciencedirect.com/science/article/pii/S2351978920313408

[47] A. Lattanzi, A. Piccininni, P. Guglielmi, M. Rossi, G. Palumbo, A fast methodology for the accurate characterization 665 and simulation of laser heat treated blanks, International Journal of Mechanical Sciences 192 (2021) 106134. doi:https: //doi.org/10.1016/j.ijmecsci.2020.106134. URL http://www.sciencedirect.com/science/article/pii/S0020740320342399

[48] L. Gambirasio, E. Rizzi, An enhanced Johnson-Cook strength model for splitting strain rate and temperature effects on lower yield stress and plastic flow, Computational Materials Science 113 (2016) 231 - 265. doi:https://doi.org/10. 1016/j.commatsci.2015.11.034

URL http://www.sciencedirect.com/science/article/pii/S0927025615007417

[49] Surajit Kumar Paul, Predicting the flow behavior of metals under different strain rate and temperature through

phenomenological modeling, Computational Materials Science 65 (2012) 91 - 99. doi:https://doi.org/10.1016/j. commatsci.2012.06.039

URL http://www.sciencedirect.com/science/article/pii/S0927025612004107

[50] Y. Lin, X.-M. Chen, A critical review of experimental results and constitutive descriptions for metals and alloys in hot working Materials \& Design 32 (4) (2011) 1733 - 1759. doi:https://doi.org/10.1016/j.matdes.2010.11.048. URL http://www.sciencedirect.com/science/article/pii/S0261306910006746

551] D. Notta-Cuvier, B. Langrand, E. Markiewicz, F. Lauro, G. Portemont, Identification of Johnson-Cook's Viscoplastic Model Parameters Using the Virtual Fields Method: Application to Titanium Alloy Ti6Al4V| Strain 49 (1) (2013) 22-45. arXiv:https://onlinelibrary.wiley.com/doi/pdf/10.1111/str.12010, doi:10.1111/str.12010

URL https://onlinelibrary.wiley.com/doi/abs/10.1111/str.12010

[52] S. Liu, A. Kouadri-Henni, A. Gavrus, Dp600 dual phase steel thermo-elasto-plastic constitutive model considering strain rate and temperature influence on fem residual stress analysis of laser welding, Journal of Manufacturing Processes 35 (2018) 407-419. doi:https://doi.org/10.1016/j.jmapro.2018.07.006 URL https://www.sciencedirect.com/science/article/pii/S1526612518308752

[53] E.-H. Lee, D.-Y. Yang, J. W. Yoon, W.-H. Yang, Numerical modeling and analysis for forming process of dual-phase 980 steel exposed to infrared local heating. International Journal of Solids and Structures 75-76 (2015) 211-224. doi:https: //doi.org/10.1016/j.ijsolstr.2015.08.014. URL https://www.sciencedirect.com/science/article/pii/S0020768315003613

[54] A.G. Holzapfel, , Nonlinear Solid Mechanics: A Continuum Approach for Engineering, John Wiley \& Sons, LTD., 2000.

[55] A. Marek, and F.M. Davis, and M. Rossi,and F. Pierron, Extension of the sensitivity-based virtual fields to large deformation anisotropic plasticity, International Journal of Material Forming 12 (3) (2019) 457-476.

[56] E.M.C. Jones, and K.N. Karlson, and P.L. Reu, Investigation of assumptions and approximations in the virtual 695 fields method for a viscoplastic material model, Strain 55 (4) (2019) e12309, e12309 10.1111/str.12309. arXiv:https: //onlinelibrary.wiley.com/doi/pdf/10.1111/str.12309, doi:10.1111/str.12309 URL https://onlinelibrary.wiley.com/doi/abs/10.1111/str.12309

[57] F. Pierron and S. Avril and V. The Tran, Extension of the virtual fields method to elasto-plastic material identification with cyclic loads and kinematic hardening. International Journal of Solids and Structures 47 (22) (2010) $2993-3010$. doi:https://doi.org/10.1016/j.ijsolstr.2010.06.022 URL http://www.sciencedirect.com/science/article/pii/S002076831000243X

[58] J.M.P. Martins, and S. Thuillier, and A. Andrade-Campos, Calibration of anisotropic plasticity models with an optimized heterogeneous test and the virtual fields method, in: Residual Stress, Thermomechanics \& Infrared Imaging and Inverse Problems, Volume 6, Springer, 2020, pp. 25-32.

705 [59] M.A. Crisfield,, Non-Linear Finite Element Analysis of Solids and Structures, Volume 1, John Wiley \& Sons, Ltd., 1991.

[60] J.C. Simo, and T.J.R. Hughes, Computational inelasticity, Springer, New York, NY, 1998. doi:https://doi.org/10. $1007 / \mathrm{b} 98904$

[61] S. Coppieters, T. Kuwabara, Identification of post-necking hardening phenomena in ductile sheet metal, Experimental mechanics 54 (8) (2014) 1355-1371. doi:https://doi.org/10.1007/s11340-014-9900-4.

710 [62] A. Brosius, N. Küsters, M. Lenzen, New method for stress determination based on digital image correlation data, CIRP Annals 67 (1) (2018) 269-272. doi:https://doi.org/10.1016/j.cirp.2018.04.026

URL https://www.sciencedirect.com/science/article/pii/S0007850618300507

[63] M. Rossi, A. Lattanzi, L. Cortese, D. Amodio, An approximated computational method for fast stress reconstruction

in large strain plasticity. International Journal for Numerical Methods in Engineering 121 (14) (2020) 3048-3065. doi: https://doi.org/10.1002/nme.6346 URL https://onlinelibrary.wiley.com/doi/abs/10.1002/nme.6346

[64] D.W. Marquardt, An algorithm for least-squares estimation of nonlinear parameters, Journal of the society for Industrial and Applied Mathematics 11 (2) (1963) 431-441. doi:10.1137/0111030

[65] I. MathWorks, Matlab 2017b (2017).

720 66] J.-H. Kim and A. Serpantié and F. Barlat and F. Pierron and M.-G. Lee, Characterization of the post-necking strain hardening behavior using the virtual fields method International Journal of Solids and Structures 50 (24) (2013) 3829 3842. doi:https://doi.org/10.1016/j.ijsolstr.2013.07.018

URL http://www.sciencedirect.com/science/article/pii/S0020768313002989

[67] S. Coppieters and J.-H. Kim and K. Denys and S. Cooreman and D. Debruyne, On complete solutions for the problem of 
diffuse necking in sheet metal Procedia Engineering 207 (2017) 2012 - 2017, international Conference on the Technology a of Plasticity, ICTP 2017, 17-22 September 2017, Cambridge, United Kingdom. doi:https://doi.org/10.1016/j.proeng. 2017.10 .1060

URL http://www.sciencedirect.com/science/article/pii/S1877705817358551

[68] M. Rossi, F. Pierron, On the use of simulated experiments in designing tests for material characterization from full-field

730 measurements International Journal of Solids and Structures 49 (3) (2012) 420-435. doi:https://doi.org/10.1016/j. ijsolstr.2011.09.025

URL https://www.sciencedirect.com/science/article/pii/S0020768311003891

[69] M. Rossi, P. Lava, F. Pierron, D. Debruyne, M. Sasso, Effect of DIC spatial resolution, noise and interpolation error on identification results with the VFM Strain 51 (3) (2015) 206-222. arXiv:https://onlinelibrary.wiley.com/doi/pdf/ 10.1111/str.12134 doi:https://doi.org/10.1111/str.12134

URL https://onlinelibrary.wiley.com/doi/abs/10.1111/str.12134

[70] L. Fletcher, F. Davis, S. Dreuilhe, A. Marek, F. Pierron, High strain rate elasto-plasticity identification using the imagebased inertial impact (IBII) test part 1: Error quantification. Strain 57 (2) (2021) e12375, e12375 10.1111/str.12375. arXiv:https://onlinelibrary.wiley.com/doi/pdf/10.1111/str.12375, doi:https://doi.org/10.1111/str.12375 URL https://onlinelibrary.wiley.com/doi/abs/10.1111/str.12375 